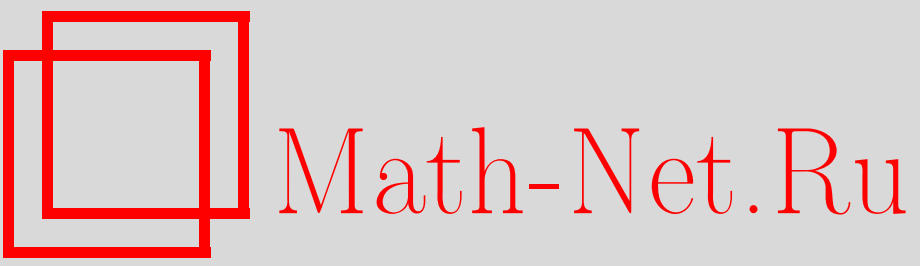

C. Ю. Доброхотов, Об эффектах интегрируемости укороченных цепочек Гюгонио-Маслова для траекторий мезомасштабных вихрей на мелкой воде, TMФ, 2000, том 125, номер 3, 491-518

DOI: https://doi.org/10.4213/tmf681

Использование Общероссийского математического портала Math-Net.Ru подразумевает, что вы прочитали и согласны с пользовательским соглашением

http://www.mathnet.ru/rus/agreement

Параметры загрузки:

IP: 54.224 .60 .19

26 апреля 2023 г., 13:08:23 
ТЕОРЕТИЧЕСКАЯ

И МАТЕМАТИЧЕСКАЯ

ФИЗИКА

Том 125, № 3

декабрь, 2000

(C) 2000 г.

С.Ю. Доброхотов*

\title{
ОБ ЭФФЕКТАХ ИНТЕГРИРУЕМОСТИ УКОРОЧЕННЫХ ЦЕПОЧЕК ГЮГОНИО-МАСЛОВА ДЛЯ ТРАЕКТОРИЙ МЕЗОМАСШТАБНЫХ ВИХРЕЙ НА МЕЛКОЙ ВОДЕ
}

\begin{abstract}
Цель этой статьи - обратить внимание специалистов на факты типа интегрируемости в задаче об описании траекторий "больших" (мезомасштабных) вихрей на мелкой воде. Согласно гипотезе Маслова такие вихри могут быть заданы с помощью решений со слабыми точечными особенностями типа квадратного корня из квадратичной формы, и тем самым такие "корневые" особые решения могут быть использованы в задаче распространения мезомасштабных вихрей в атмосфере (тайфунов и циклонов). Таким решениям с необходимостью соответствуют бесконечные системы обыкновенных дифоференциальных уравнений (цепочки) для тейлоровских коэффициентов функций, задающих решения в окрестности особенности. Достаточно разумное замыкание "вихревой цепочки" для системы уравнений мелкой воды дает систему из 17 нелинейных уравнений. Показано, что эта система в случае постоянной силы Кориолиса сводится к хорошо известному уравнению Хилла, а в случае силы Кориолиса, зависящей от широты местности, - к "почти" уравнениям физического маятника. Эти факты позволяют в грубом приближении достаточно явно описать возможные траектории мезомасштабных вихрей и, в частности, провести аналогию между ними и колебаниями на упругой нити вращающегося твердого тела.
\end{abstract}

\section{1. ВВЕДЕНИЕ. ПОСТАНОВКА ЗАДАЧИ И РЕЗУЛЬТАТЫ}

Около 20 лет назад Маслов [1] обнару жил, что многие квазилинейные гиперболические системы, включая, в частности, уравнения гидродинамики, допускают лишь конечное число особых решений, сохраняющих на некоторых временных промежутках свою структуру и находящихся в "общем положении". К числу таких решений относятся ударные волны, "бесконечно узкие" солитоны и уединенные вихри. Разумеется, отбор возможных особенностей обусловлен наличием нелинейных членов: для линейных гиперболических систем, как известно, решения, по крайней мере в малом, наследуют любой тип особенности начального условия. Согласно развитой в [1] концепции, несмотря на различие физической природы описываемых объектов, с математической точки зрения такие особые решения имеют много обшего. Одной из обших важных характеристик этих решений является наличие неизвестных ранее бесконечных систем (цепочек) обык-

\footnotetext{
* Институт проблем механики РАН, Москва, Россия. E-mail: dobr@ipmnet.ru
} 
новенных дифференциальных уравнений, с необходимостью возникающих и определяюших в некотором смысле динамику решений. Для ударных волн первое из уравнений соответствуюших цепочек есть хорошо известное условие Гюгонио (см., например, [2-5]), а остальные представляют собой поправки к нему. Достаточно подробное исследование таких цепочек для ударных волн уравнений газодинамики и солитонов было проведено довольно давно (см. [6-8] и библиографию в $[9,10])$. Для вихревых (точечных) особенностей аналогичные цепочки до недавнего времени почти не изучались.

В теории нелинейных процессов хорошо известна система уравнений мелкой воды простейшая двумерная эволюционная модель, описываюшая нелинейные бездисперсионные невязкие процессы. Она широко используется не только в теории волн на воде, но и в ряде других областей физики и механики. В частности (это важно для данной работы), она применяется для “грубого" моделирования движения (но не образования и гибели) мезомасштабных планетарных вихрей (тайфунов и циклонов [11-16]). С учетом вращения Земли и зависимости силы Кориолиса от параллели в приближении $\beta$-плоскости эта система имеет вид

$$
\frac{\partial \eta}{\partial t}+\nabla(\eta \mathbf{u})=0, \quad \frac{\partial \mathbf{u}}{\partial t}+(\mathbf{u}, \nabla) \mathbf{u}-\omega \mathbf{T} \mathbf{u}+\nabla \eta=0
$$

Здесь $x=\left(x_{1}, x_{2}\right) \in \mathbb{R}^{2}, \mathbf{u}-2$-мерньй вектор скорости, $\eta>0$ - высота превьшения свободной поверхности в теории поверхностных волн или геопотенциал в атмосфере,

$$
\mathbf{T}=\left(\begin{array}{cc}
0 & 1 \\
-1 & 0
\end{array}\right)
$$

- матрица поворота на $90^{\circ}$,

$$
\nabla=\left(\frac{\partial}{\partial x_{1}}, \frac{\partial}{\partial x_{2}}\right),
$$

$\omega / 2$ - частота Кориолиса на $\beta$-плоскости: $\omega=\widetilde{\omega}+\beta x_{2}, \widetilde{\omega}=2 \Omega \sin \Theta, \beta=2 \Omega \cos \Theta / R$, где $\Theta$ определяет широту точки на сфере (Земле), к которой проводится касательная $\beta$-плоскость, $\Omega$ - частота вращения, $R$ - средний радиус Земли. Считается, что координаты на $\beta$-плоскости отсчитываются от точки касания, причем изменение координаты $x_{1}$ соответствует движению по параллели, а $x_{2}-$ по меридиану ${ }^{1)}$. Сушественно, что система (1) обладает свойством “сжимаемости” (в силу уравнения непрерывности), несмотря на то что исходные полные уравнения гидро- и газодинамики, из которых она выводится в результате некоторых асимптотических процедур, могут быть бездивергентными $^{2)}$. Для нас факт сжимаемости оказывается весьма сушественным: как известно, условия Гюгонио можно написать лишь в сжимаемых средах.

Вихревые (слабые) особые решения, указанные в [1], имеют вид

$$
\mathbf{w}=\mathbf{f}(x, t)+\mathbf{g}(x, t), \quad \mathbf{g}(x, t)=g(x, t) \sqrt{S(x, t)} .
$$

\footnotetext{
1) В задаче о движениях тайфунов мы измеряем время в часах, расстояние в километрах и в качестве угла $\Theta$ берем угол $30^{\circ}$. Тогда параметр $\beta$ оказывается равным $0.00007 \mathrm{kм}^{-1}$ час $^{-1}$.

2) Этот важный и любопытный факт особо обсуждается в [17], § 108, гл. Х.
} 
Здесь $\mathbf{w}$ - вектор-функция с компонентами $\mathbf{u}_{1}, \mathbf{u}_{2}, \eta$; “фон" $\mathbf{f}(x, t)={ }^{t}(v(x, t), w(x, t)$, $\rho(x, t))$ и "векторная амплитуда" $g(x, t)={ }^{t}\left(U_{1}(x, t), U_{2}(x, t), R(x, t)\right)$ - гладкие векторфункции; "фаза" $S(x, t)$ - скалярная функция, в первом приближении совпадаюшая с “асимметричной" положительной квадратичной формой:

$$
S(x, t)=\frac{1}{2}\left(x-X(t), S^{\prime \prime}(X(t))(x-X(t))\right)+O\left(|x-X(t)|^{3}\right) ;
$$

ее центр - гладкая вектор-функция $X(t)={ }^{t}\left(X_{1}(t), X_{2}(t)\right)$ - определяет траекторию $\Gamma$ движения вихревой (слабой) точечной особенности, и $S^{\prime \prime}(x)$ - матрица вторых производных (гессиан) от функции $S(x, t)$ с различными при каждом $t$ собственными значениями. В написанных выше выражениях индекс $t$ вверху слева означает транспонирование. В работе [1] была высказана гипотеза о том, что именно особые вихревые решения ${ }^{3)}$ вида (2) системы (1) соответствуют мезомасштабным вихрям в атмосфере. Тем самым отвечаюшие им "вихревые" цепочки в некотором приближении могут моделировать движение "глаза" тайфуна. Подчеркнем, что мы не занимаемся вопросами возникновения, диссипации, детального и полного описания структуры вихрей в атмосфере - для этого требуется рассмотрение более сложных уравнений, чем (1). Мы также не занимаемся вопросами сушествования вихрей и их взаимодействия, а рассматриваем задачу, которую кратко можно сформулировать так: если у системы уравнений (1) есть уединенный вихрь с некоторыми спечиальными (но достаточно разумными) физическими свойствами, то по какой траектории он будет двигаться и какова его форма в окрестности иентра? Немаловажным фактом в пользу выбора тайфунов в качестве примеров вихрей, приближенно описываемых системой (1), является также возможность сравнения полученных теоретических результатов с реальными траекториями.

Исследование указанных цепочек, описываюших распространение вихревых особенностей для системы уравнений "мелкой воды” с переменной силой Кориолиса, начатое сравнительно недавно [20], выявило [9, 21, 22] совершенно неожиданные свойства типа "интегрируемости". В конечном итоге в предположении, что частота Кориолиса есть константа $(\beta=0)$, эти свойства приводят в некотором приближении к описанию траекторий мезомасштабных вихрей с помошью семейства (линейных) уравнений Хилла

$$
\frac{d^{2} \psi}{d \Phi^{2}}+\left(\lambda+\frac{1}{c^{4}} \operatorname{Re}\left(\left(\alpha_{1} \alpha_{2}-\alpha_{0} \overline{\alpha_{2}}\right) e^{i \Phi}+\frac{3}{2} \alpha_{0} \alpha_{1} e^{2 i \Phi}\right)\right) \psi=0
$$

где $\alpha_{0}, \alpha_{1}, \alpha_{2}$ - комплексные, $\lambda, c$ - вешественные параметры, характеризующие траектории, черта означает комплексное сопряжение. Удалось также связать некоторые свойства траекторий со спектральными характеристиками и свойствами устойчивости уравнения (3). Такая связь с дополнительным предположением о достаточной "гладкости" траекторий (т.е. отсутствии у них больших петель) позволила, в свою очередь, выделить важные подсемейства "гладких" траекторий, которые также неожиданно (и с

\footnotetext{
${ }^{3)}$ Соображение, что именно особые решения соответствуют мезомасштабным вихрям в атмосфере, используется достаточно часто (см., в частности, $[18,19])$.
} 


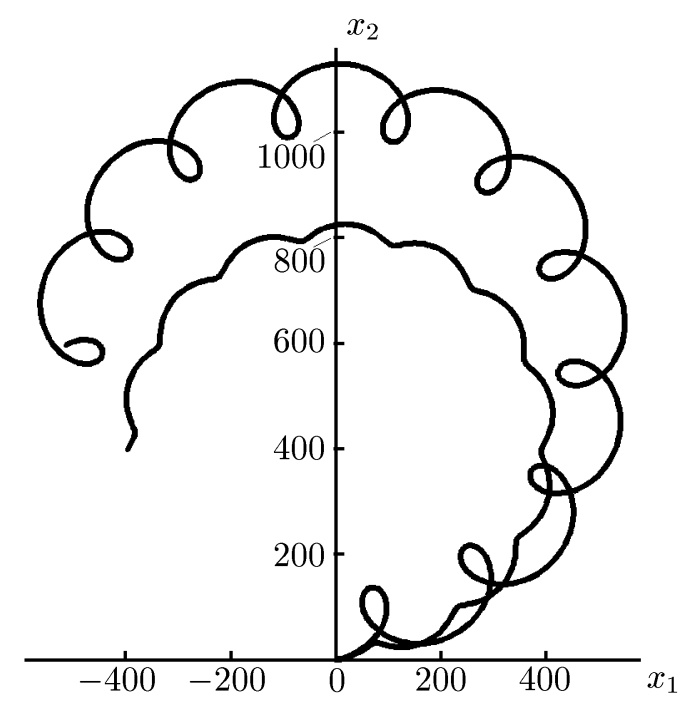

Рис. 1

необходимостью) описываются исключительно простыми формулами и определяют на плоскости $\left(x_{1}, x_{2}\right)$ циклоидальные кривые (рис. 1$)$.

Именно, если считать $X(t)$ комплексным вектором плоскости, то одно из трех таких семейств имеет вид

$$
X=A+B e^{i \omega_{0} t}+C e^{i\left(p-\omega_{0}\right) t}
$$

где $A, B, C$ - комплексные, $p, \omega_{0}$ - вешественные параметры, характеризующие траекторию, при этом $\omega_{0}$ - частота Кориолиса, $p$ - третья компонента ротора скорости на траектории. Если частота $p-\omega_{0}$ мала, движение по траектории складывается из медленного движения по “большой" окружности (с этой угловой скоростью) и быстрого - с "собственной" кориолисовой частотой $\omega_{0}$. Весьма любопытной оказывается связь направления движения по приведенным траекториям с зонами устойчивости уравнения Хилла (3). Траектория (4) соответствует нулевым значениям параметров $\alpha_{1}, \alpha_{2}$ и значению $\lambda$, лежашему в окрестности точки $1 / 4$, т.е. в окрестности “следа" первой зоны неустойчивости уравнения Хилла. Если $\lambda<1 / 4$, то $p>\omega_{0}$ и движение происходит по часовой стрелке, если $\lambda>1 / 4$, то $p<\omega_{0}$ и движение происходит против часовой стрелки. Таким образом, в отсутствие “ $\beta$-эффекта" направление движения является инвариантом, связанньцм с первой и второй зонами устойчивости уравнения Хилла (3) (рис. 2).

Чтобы пояснить неожиданность появления уравнения Хилла (3) и появления траекторий (4), приведем систему из 17 обыкновенных дифференциальных уравнений ("оборванную" или "укороченную” цепочку Гюгонио-Маслова), которая точно сводится к (3) и компоненты $X_{1}(t), X_{2}(t)$ некоторого частного решения которой совпадают с $(4)$. Эти 


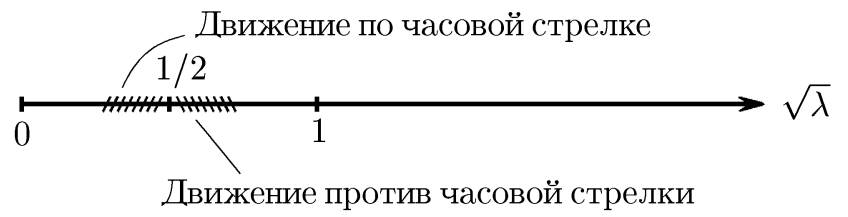

Рис. 2

уравнения пишутся для нулевых, первых и вторых тейлоровских коэффициентов ${ }^{4)}$ разложения фона $\mathbf{f}(x, t)={ }^{t}(v(x, t), w(x, t), \rho(x, t))$ в окрестности $X(t)$. Их аккуратный вывод требует нетривиальных и объемных выкладок [9], мы дадим здесь лишш основные идеи этого вывода.

Обозначим точкой дифференцирование по времени и через

$$
G_{m_{1} m_{2}}=\frac{1}{m_{1} ! m_{2} !} \frac{\partial^{m_{1}+m_{2}} G}{\partial x_{1}^{m_{1}} \partial x_{2}^{m_{2}}}(X(t), t)
$$

- тейлоровские коэффициенты гладких скалярных и векторных функций $G(x, t)$. Будем писать $\rho_{0}(t), \omega_{0}(t), V(t)$ вместо $\rho_{00}, \omega_{00}$ и $u_{00}$. Сначала в предположении, что “фоновые" функции $v(x, t), w(x, t), \rho(x, t)$ заданы, исследуется вопрос, когда решения вида (2) существуют и одновременно старшая часть сингулярной составляющей восстанавливается через $v, w, \rho$. Оказывается, для сушествования такого сингулярного решения необходимо, чтобы

1) траектория особенности $X(t)$ была “вморожена" в поле скоростей и (которое на траектории совпадает с полем скоростей $u$ фона):

$$
\dot{X}(t)=u(X(t), t) \equiv \mathbf{u}(X(t), t) \equiv V(t) \Longleftrightarrow \dot{X}_{1}=V_{1}, \quad \dot{X}_{2}=V_{2}
$$

2) на траектории $X(t)$ для комплексных скоростей $\mathbf{v}(x, t)=v(x, t)+i w(x, t)$ были выполнены условия Коши-Римана

$$
\begin{aligned}
& \frac{\partial v}{\partial x_{1}}=\frac{\partial w}{\partial x_{2}} \equiv q(t) \equiv \frac{1}{2} \operatorname{div} u, \\
& \frac{\partial v}{\partial x_{2}}=-\frac{\partial w}{\partial x_{1}} \equiv p(t) \equiv-\frac{1}{2} \operatorname{rot}_{3} u
\end{aligned}
$$

при $x=X(t)$ и

$$
\rho_{20}=\rho_{02}+\frac{\beta V_{1}}{2}, \quad \rho_{11}=\frac{\beta V_{2}}{2} .
$$

Соотношения (5)-(7) представляют собой аналог условий Гюгонио (и поправок к ним) для вихрей. При этом на соотношения (5) можно смотреть как на уравнения, а на (6),

\footnotetext{
4) Эти коэффициенты необходимы, поскольку именно с такими номерами надо найти коэффициенты разложения функции $S$, чтобы определить старшую часть негладкой составляющей решения.
} 
(7) - как на уравнения связи ${ }^{5)}$, налагаемой сингулярностью (мезомасштабным вихрем) на "фон”.

Далее, отбросив из решения "негладкую часть" $\mathrm{g}(x, t)$, нужно подставить в уравнения (2) тейлоровские разложения “фона” в точках траектории $X(t)$ и приравнять нулю коэффициенты при нулевых, первых и вторых степенях $x_{j}-X_{j}(t)$. В дополнение к (5) это даст 18 соотношений, связываюших коэффициенты $u_{i j}, w_{i j}, \rho_{i j}$ с номерами $i$, $j, i+j \leqslant 3$, и производные $\dot{u}_{i j}, \dot{w}_{i j}, \dot{\rho}_{i j}$ уже с номерами $i, j, i+j \leqslant 2$. Кроме того, имеем равенство $\omega_{0}=\widetilde{\omega}+\beta X_{2}(t)$, которое удобно заменить на равенство $\dot{\omega}_{0}=\beta V_{2}(t)$. В результате получаем 21 соотношение. Четыре "уравнения связи" (6), (7) сокрашают их количество до 17. Эти соотношения, однако, не образуют замкнутую систему уравнений - количество неизвестных в ней превышает количество уравнений. Для их замыкания мы отбрасываем ${ }^{6}$ коэфффициенты $u_{i j}, w_{i j}, \rho_{i j}$ с номерами $i, j, i+j=3$. Это вместе с (5) и приводит к упомянутой системе из 17 нелинейных уравнений, в которой, за исключением постоянной (параметра) $\beta$, все переменные являются неизвестными:

$$
\begin{gathered}
\dot{V}_{1}+\omega_{0} V_{2}+\rho_{10}=0, \quad \dot{V}_{2}-\omega_{0} V_{1}+\rho_{01}=0, \quad \dot{\rho}_{0}+2 q \rho_{0}=0, \quad \dot{\omega}_{0}-\beta V_{2}=0, \\
\dot{q}-p^{2}+q^{2}+\omega_{0} p+2 r+\frac{\beta V_{1}}{2}=0, \quad \dot{p}+2 p q-\omega_{0} q-\frac{\beta V_{2}}{2}=0, \\
\dot{\rho}_{10}+3 q \rho_{10}-p \rho_{01}+\rho_{0}\left(w_{11}+2 v_{20}\right)=0, \\
\dot{\rho}_{01}+3 q \rho_{01}+p \rho_{10}+\rho_{0}\left(v_{11}+2 w_{02}\right)=0 \\
\dot{r}+4 q r+\frac{1}{2} \rho_{10}\left(3 v_{20}+w_{11}+v_{02}\right)+\frac{1}{2} \rho_{01}\left(v_{11}+3 w_{02}+w_{20}\right)=f^{r}, \\
\dot{v}_{20}+3 q v_{20}-\omega_{0} w_{20}-p\left(v_{11}-w_{20}\right)=f_{20}^{v}, \\
\dot{v}_{11}+3 q v_{11}-\omega_{0} w_{11}-p\left(2 v_{02}-2 v_{20}-w_{11}\right)+\beta p=f_{11}^{v}, \\
\dot{v}_{02}+3 q v_{02}-\omega_{0} w_{02}+p\left(v_{11}+w_{02}\right)-\beta q=-f_{02}^{v}, \\
\dot{w}_{20}+3 q w_{20}+\omega_{0} v_{20}-p\left(w_{11}+v_{20}\right)=f_{20}^{w}, \\
\dot{w}_{11}+3 q w_{11}+\omega_{0} v_{11}-p\left(-2 w_{20}+2 w_{02}+v_{11}\right)+\beta q=f_{11}^{w}, \\
\dot{w}_{02}+3 q w_{02}+\omega_{0} v_{02}+p\left(w_{11}-v_{02}\right)+\beta p=f_{02}^{w} .
\end{gathered}
$$

Мы обозначили $r=\rho_{20}-\beta V_{1} / 4 \equiv \rho_{02}+\beta V_{1} / 4$ и включили в систему отбрасываемые в результате замыкания правые части $f^{r}, f_{20}^{v}$ и т.д., которые опишем позднее. Именно эта система с $f^{r}=f_{i j}^{v}=f_{i j}^{w}=0$ и $\beta=0$ редушируется к уравнениям Хилла (3) (параметры $\lambda, \alpha_{0,1,2}, c$ суть часть ее констант интегрирования), а формулы (4) определяют $X$-компоненты одного из ее (точных) решений. Основная сложность в выводе системы (8) содержится в получении соотношений (5)-(7). Они находятся с помошью соображений, аналогичных соображениям, используемым в методе ВКБ и в разложениях по “гладкости" [23, 24]. Подчеркнем, что вывод условий (5)-(7) сушественно использует предположение об "асимметрии вихря" (матрицы $S^{\prime \prime}(X(t), t)$ ) и наличие нелинейности.

\footnotetext{
5) Такая интерпретация соотношений (6), (7) подсказана Г. Г. Черным.

6) Некоторые аргументы, поясняющие разумность такого замыкания, мы приводим ниже в разделах 2-4.
} 
Вывод соотношений (8), по сушеству, заключается в последовательном дифференцировании исходной системы (точнее, ее гладкой составляюшей) с учетом (5)-(7) и вычислении результата в точках траектории $x=X(t)$. При этом требуется лишь проведение громоздких выкладок.

Включение параметра $\beta$ меняет ситуацию достаточно сильно, если времена сравнимы с $1 /(\beta d)$ ( $d$ - характерное расстояние), что и имеет место в задаче о тайфунах и вполне согласуется с мнением специалистов о существенном влиянии " $\beta$-эффекта" на траектории (см., например, $[14,16])$. Параметр $\beta$ в изучаемой системе является малым, и данная система относится к типичным адиабатически возмущенным задачам, в которых применяются методы осреднения. При этом константы интегрирования системы с $\beta=0$ становятся медленно меняющимися функциями. Мы уже отмечали, что разумные с точки зрения задачи о тайфунах предположения о гладкости траекторий и малом изменении геопотенциала выделяют среди решений системы $(5),(8)$ решения с малой частотой $p-\omega_{0}$. Поэтому исследование эволюции под действием “ $\beta$-эффекта” именно таких решений (которым при $\beta=0$ соответствуют траектории (4)) вызывает особый интерес. Поскольку частота $p-\omega_{0}$ является малой, то речь здесь идет о частичном осреднении (см. [25-27]). Заметим, что поскольку $\lambda$ (так же как и другие параметры в уравнении Хилла) уже становится “медленно меняющейся функцией”, то появляется возможность перехода из одной зоны устойчивости в другую (такой эффект в физике твердого тела называется пробоем Зинера), что может привести к изменению направления движения, определяемого равенством (4), и появлению зигзагов у траекторий.

Еще один интересный и также неожиданный, но, видимо, не случайный факт состоит в том, что осредненные уравнения для такого режима оказываются интегрируемыми в квадратурах и близки к уравнениям физического маятника! Именно осредненные уравнения сводятся к системе из двух уравнений для частоты $\omega_{0}(t)$ и угла $\psi(t)$, задающего направление скорости движения вихря по траектории $V=\dot{X}$. Эти уравнения зависят элементарным образом от $\beta$ и параметров (констант интегрирования) $\omega_{0}(0), \psi(0)$, $R, \Omega, c$ (смысл трех последних мы проясним позднее). Для упрошения обозначений эту зависимость у соответствующих решений мы, как правило, будем опускать. Приведем эту систему сначала в предположении, что $c V^{2} \gg \omega_{0}$. Тогда зависимость от $\beta$ и $R$ входит в нее в виде произведения, а зависимость от $c$ вообше пропадает, и мы имеем

$$
\dot{\omega}_{0}=\beta R K\left(\omega_{0}, \Omega\right) \sin \psi, \quad \dot{\psi}=N\left(\omega_{0}, \Omega\right),\left.\quad \omega_{0}\right|_{t=0}=\omega_{0}(0),\left.\quad \psi\right|_{t=0}=\psi(0) .
$$

Здесь

$$
N\left(\omega_{0}, \Omega\right) \equiv p-\omega_{0}=\omega_{0}\left(1-\frac{3}{2} \sqrt{\frac{\omega_{0}}{\Omega}}\right), \quad K\left(\omega_{0}, \Omega\right)=2 \sqrt{3 \Omega}\left(1-\sqrt{\frac{\omega_{0}}{\Omega}}\right)^{\frac{3}{2}}
$$

При этом скорость $V$, траектория $X$, переменная $p$, геопотенциал $\rho_{0}$ и $\lambda$ восстанавливаются через $\omega_{0}(t) \quad\left(0<\omega_{0}(t)<\Omega\right)$ и $\psi(t)$ с помошью формул

$$
\begin{gathered}
V=V(t)=K\left(\omega_{0}, \Omega\right) R e^{i \psi}, \quad X(t)=X^{0}+\int_{0}^{t} V(\eta) d \eta \\
p=\omega_{0}\left(2-\frac{3}{2} \sqrt{\frac{\omega_{0}}{\Omega}}\right), \quad c \rho_{0}=3 \omega_{0}\left(1-\sqrt{\frac{\omega_{0}}{\Omega}}\right), \quad \lambda^{-\frac{1}{2}}=6\left(1-\sqrt{\frac{\omega_{0}}{\Omega}}\right) .
\end{gathered}
$$

6 Теоретическая и математическая физика, т. 125, № 3, 2000 г. 
Укажем на следующий любопытный факт: "резонансное" равенство $p=\omega_{0}$ имеет место при $\omega_{0}=4 \Omega / 9$, когда (одновременно) функция $\rho_{0}=\rho_{0}\left(\omega_{0}\right)$ имеет максимум (а следовательно, функция $\rho_{0}$ на "резонансном" инвариантном многообразии меняется слабо), а "спектральный параметр" уравнения Хилла $\lambda=\lambda\left(\omega_{0}\right)$ принимает значение $1 / 4$, т.е. $\lambda(4 \Omega / 9)$ является “следом" первой зоны неустойчивости уравнений Хилла (8) при $Q \rightarrow \lambda$ (см. рис. 3а).

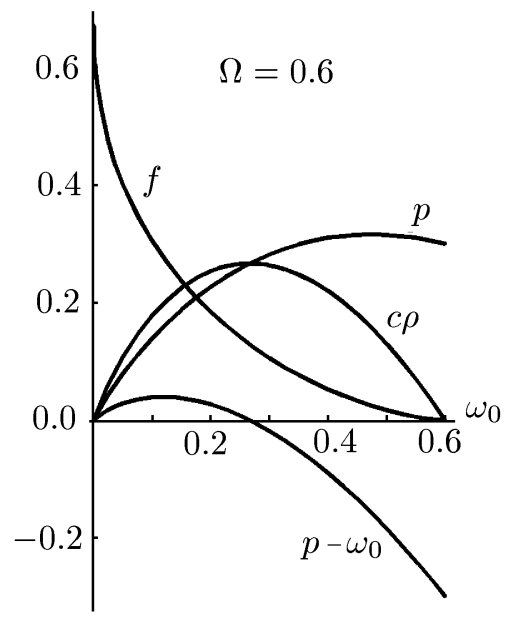

a

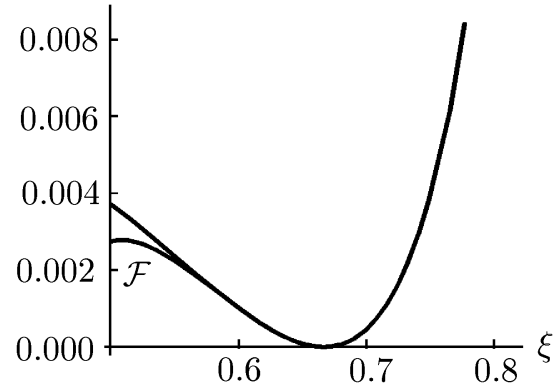

б

Рис. 3

Вернемся к системе (8). Мы уже отмечали, что она интегрируема в квадратурах, ее интеграл также определяется с помощью әлементарных функиий (см. графики функции $\mathcal{F}$ и ее тейлоровского разложения до четвертого члена на рис. $3 б):$

$$
\begin{aligned}
& \mathcal{F}\left(\frac{\omega_{0}}{\Omega}\right)+\frac{\beta R \sin ^{2}\left(\frac{\psi}{2}\right)}{4 \sqrt{3}(\Omega)^{2}}=\text { const, } \\
& \mathcal{F}(\xi)=\frac{32-16 \sqrt{\xi}-4 \xi-2 \xi^{\frac{3}{2}}-3 \xi^{2}}{14 \sqrt{3}(1-\sqrt{\xi})^{\frac{1}{2}}}-\frac{248}{189}= \\
& =\frac{3}{16}\left(\xi-\frac{4}{9}\right)^{2}+\frac{81}{128}\left(\xi-\frac{4}{9}\right)^{3}+O\left(\left(\xi-\frac{4}{9}\right)^{4}\right) \text {, }
\end{aligned}
$$

и ее фазовый портрет на плоскости $\left(\omega_{0}, \psi\right)$ (рис. 4$)$ почти совпадает с хорошо известным (см., например, [28]) фазовым портретом траекторий физического маятника.

Этот факт позволяет провести простую классификацию траекторий вихрей указанного режима, которую мы приведем в разделе 8 .

Естественным образом возникают следующие вопросы. Что приводит к особым решениям вида (2) и почему эти решения выделяются из других особых решений? Вьше 


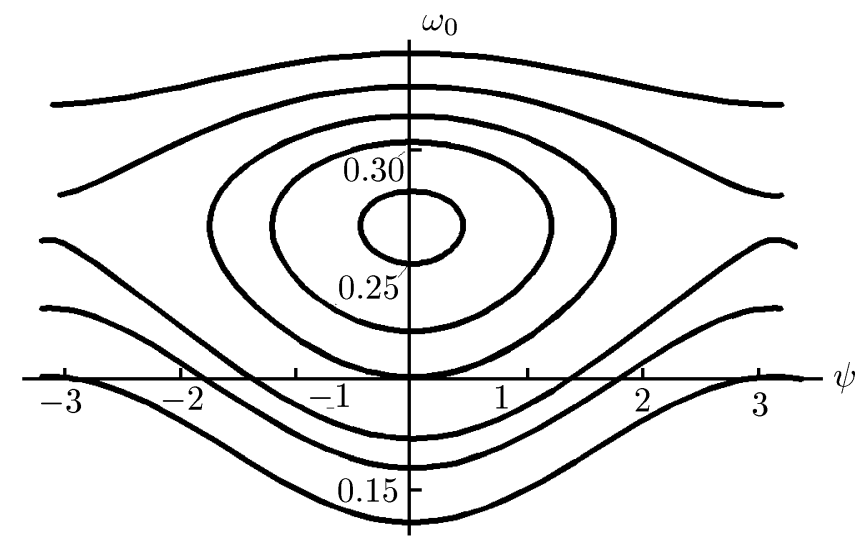

Рис. 4

мы привели некоторые пояснения, касаюшиеся вывода уравнений (5)-(8), но остается еше вопрос об их редукции к уравнению Хилла (3) и к системе (9). Ответы на эти вопросы требуют привлечения соображений из разных разделов математической физики, а также проведения трудоемких и объемных аналитических вычислений. Эти вычисления были проделаны нами в полном объеме в работах [9]. Цель настояшей работы - изложение идейных соображений, приводяших к ответам на сформулированные вопросы, а также их иллюстрация, опирающаяся на достаточно простые формулы. Кроме того, мы сформулируем ряд новых вопросов, порожденных исследованиями [9], представляюших на наш взгляд интерес для спешиалистов в области математической физики, механики, физики атмосферы и океана. Подчеркнем, что, несмотря на математическую сложность вывода уравнений, сами окончательные результаты неожиданно оказались настолько простыми, что могут быть доступны для понимания даже неспециалистов. Выведенные же уравнения "типа физического маятника", приближенно описываюшие возможные траектории мезомасштабных вихрей, по всей видимости, могут быть использованы в задаче восстановления и предсказания траекторий тайфунов по их уже известной, например с помощью наблюдения со спутников, части.

\section{2. ОСОБЫЕ РЕШЕНИЯ КВАЗИЛИНЕЙНЫХ ГИПЕРБОЛИЧЕСКИХ СИСТЕМ}

Мотивы появления особых решений вида (2) обсуждались в работах $[1,9,20,21,29]$, мы приводим их для полноты изложения в сжатой форме. Особые решения квазилинейных гиперболических систем, о которых шла речь в самом начале статьи, включая решения (2), могут быть описаны формулой, похожей на "нелинейные ВКБ- или уиземовские" решения или "искаженные" простые волны Римана (см., например, $[3,4,5]$ )

$$
\mathbf{w}=\mathbf{f}(x, t)+\mathbf{g}(x, t), \quad \mathbf{g}(x, t)=g(x, t) F(S(x, t)),
$$


где $\mathbf{w}$ - векторная (или скалярная) функция; $x \in \mathbb{R}^{n} ; F(\tau)$ - некоторая скалярная функция, гладкая вне множества $\tau=0$ и имеюшая особенность в точке $\tau=0$; фаза $S(x, t)$, векторные (или скалярные) “фон” $\mathbf{f}(x, t)$ и “амплитуда" $g(x, t)$ - гладкие функции. Таким образом, структура особых составляющих оказывается одинаковой для всех компонент вектора решения. Особенность может определять, например, разрыв 1-го рода (в этом случае мы имеем ударные волны) или даже быть непрерывной и один раз дифференцируемой функцией - тогда мы имеем слабые разрывы. Ясно, что особенности функции $\mathbf{w}(x, t)$ определяются нулями $X(t)$ функции $S(x, t)$. Например, для ударных волн в одномерном $(n=1)$ скалярном случае $F=\Theta(\tau)$, где $\Theta(\tau)$ - функция Хевисайда, $S=x-X(t)$, а для "бесконечно узкого солитона", движущегося по фону $\mathbf{f}(x, t)$, по-прежнему $S=x-X(t)$, но $F=\operatorname{Sol}(\tau)$, где $\operatorname{Sol}(\tau)=(0$ при $\tau \neq 0$ и 1 при $\tau=0)$. Обобшенные решения второго типа с точки зрения пространства $L_{2}$ вообше равны фону $\mathbf{f}(x, t)$, однако они оказываются вполне разумными, если правильно определить скалярное произведение и пространство основных функций. Они, например, являются пределами солитонных решений уравнения Кортевега-де Фриза с дисперсией $\varepsilon^{2}$ при $\varepsilon \rightarrow 0$. Ясно, что функция (2) также имеет структуру (13), функция $F=\sqrt{\tau}$.

Как уже говорилось, для многих физически разумных квазилинейных гиперболических уравнений почти все возможные особенности исчерпываются приведенными выше структурами, если предположить, что решения (13) удовлетворяют следующим условиям "обшего положения". Во-первых, они являются "структурно самоподобными": это означает, что если они имели форму (13) с заданной функцией $F(\tau)$ в некоторый момент времени $t_{0}$, то такая зависимость от $\tau$ сохраняется и при временах $t>t_{0}$, по крайней мере на не очень больших промежутках $t-t_{0}$. Во-вторых, они обладают свойствами "структурной устойчивости" : малое изменение начальных значений для функций $S\left(x, t_{0}\right), \mathbf{f}\left(x, t_{0}\right), g\left(x, t_{0}\right)$ (не меняющих структуру особенности по $\tau$ функции $F$ ), а также коэффициентов исходного уравнения не приводит к изменению структуры функции $\mathbf{w}$.

Поясним сказанное на примере (слабых) точечных особенностей вида (2). Естественно было бы рассмотреть решения (13) с функцией $F(\tau)$ более обшего вида, например с $F(\tau)=\tau^{\alpha}$, где $\alpha>0$ - нецелое число. Или в еше более общей ситуации можно предположить, что $F(\tau)$ - непрерывная функция для всех $\tau \geqslant 0$ и гладкая функция при $\tau>0$, и, кроме того, $F(0)=0$ и $F_{\tau} \rightarrow \infty$ при $\tau \rightarrow+0$. Относительно функции $S(x, t)$ будем считать, что $S(x, t) \geqslant 0$ и что равенство $S(x, t)=0$ при каждом $t$ имеет место лишь в единственной точке $x=X(t) \equiv\left(X_{1}(t), X_{2}(t)\right)$. Эти точки и образуют траекторию особенности $\Gamma=\{x=X(t), 0 \leqslant t \leqslant T\}, T$ - некоторый промежуток. Условия "общего положения" приводят к следуюшему требованию. На траектории особенности Г (положительная) матрица вторых производных

$$
\left.\left\|\frac{\partial^{2} S}{\partial x_{i} \partial x_{j}}\right\|\right|_{\Gamma}=\left.\operatorname{Hess} S\right|_{\Gamma}
$$

невырожденна и имеет разные собственные значения, а разложения компонент вектора $g$ по степеням $(x-X(t))$ начинаются с минимально возможных степеней. (Для системы уравнений (1) оказывается, что если решения (2) сушествуют и сформулированные предположения на $S(x, t)$ выполнены в момент времени $t=t_{0}$, то они выполнены и 
в последующие моменты времени.) Таким образом, $S$ по переменным $x$ с точностью до членов более высокого порядка малости есть положительная квадратичная форма с различными собственными значениями и центром в точках траектории $Г$ движения особенности. Мы имеем слабую “точечную” особенность: сами функции $\mathbf{u}=\left(\mathbf{u}_{1}, \mathbf{u}_{2}\right)$ и $\eta$ непрерывны, более того, равны нулю в особых точках - точках $X(t)$ траектории $\Gamma$, разрывными являются некоторые производные этих функций в точках $X(t)$ (именно такое поведение $\mathbf{w}$ служит аргументом при использовании таких особых решений для моделирования тайфунов $\left.{ }^{7)}\right)$. Тогда оказывается, что у системы уравнений мелкой воды решение вида (13) можсет существовать, только если $F=\sqrt{\tau}[1,9,29]$.

Сказанное не означает, что соответствуюшие уравнения не допускают частных, например радиально симметричных по $x-X(t)$, особых решений, отличных от приведенных выше, но они, по-видимому, быстро разрушаются под действием малых возмушений 8 )

Решения вида (13) представляют собой обобщенные функции, поэтому методы их исследования относятся к кругу вопросов, связанных с построением алгебр обобщенных функций и их использованием в нелинейных уравнениях (см. [10, 30-32] и библиографию в $[9,10,33])$.

\section{3. "ВИХРЕВЫЕ" ЦЕПОЧКИ ГЮГОНИО-МАСЛОВА И ВОССТАНОВЛЕНИЕ ОСОБЫХ РЕШЕНИЙ ПО ПАРАМЕТРАМ ТРАЕКТОРИИ}

Как уже отмечалось, к числу основных результатов работы [1] относится выявление возможности описания решений вида (2) с помошью бесконечномерных систем (цепочек) обыкновенных дифференциальных уравнений для коэффициентов рядов Тейлора разложения в окрестности разрыва Г (гладких ${ }^{9)}$ ) “фазы” $S(x, t)$, фона $\mathbf{f}(x, t)$, “амплитуды" $\mathbf{g}(x, t)$. Для ударных волн первые из этих уравнений представляют собой хорошо известные условия Гюгонио, а остальные - поправки к ним. Поскольку впервые эти цепочки уравнений появились в [1], мы называем их иепочками Гюгонио-Маслова. Они $c$ необходимостью возникают для решений вида (2) и оказываются незамкнутыми в том смысле, что первые $n$ уравнений содержат более чем $n$ неизвестных, и поэтому их решение не приводит, вообще говоря, к однозначному определению положения особенности. Тем не менее идея использования некоторого конечномерного замыкания таких цепочек для описания динамики особенностей хотя бы на каких-нибудь временных отрезках является весьма привлекательной. Замыкание возможно, если известны неко-

\footnotetext{
7) Поскольку в “глазе тайфуна" скорость ветра оказывается сравнительно небольшой.

8) Вообще привлечение соображений "общего положения" с точки зрения общепринятых подходов в математической физике приводит к несколько парадоксальной ситуации: естественно было бы начинать исследовать решения в конкретной простой ситуации, каковая, в частности, представляется радиально-симметричными случаями. Однако используемые грубые рассуждения про траектории особенностей в этом случае позволяют сказать существенно меньше, чем в случае "общего положения".

${ }^{9)}$ Напомним, что вся негладкость решения (2) содержится в $F(\tau)$.
} 
торые глобальные свойства решения или заданы какие-либо дополнительные предположения (например, о малости амплитуды разрыва [6]). Напомним, что (незамкнутые) цепочки дифференциальных уравнений хорошо известны в механике сплошных сред и математической физике, например, цепочка ББГКИ в статистической физике, моментные цепочки в статистической гидромеханике, цепочки Тоды в теории солитонов и т.д. Также хорошо известны различные связанные с ними проблемы, в том числе и проблемы их замыкания, интегрирования и т.д. Их решение оказывается далеко не простым и часто приводит к важным результатам. Например, процедура замыкания цепочки ББГКИ приводит к кинетическому уравнению Больцмана.

Для цепочек Гюгонио-Маслова, возникающих при описании ударных волн простейшего нелинейного уравнения Хопфа $v_{t}+v v_{x}=0$, в работах Прасада и Равиндрана был применен и дал весьма хорошие результаты [34] способ замыкания ("обрьв"), основанньй на приравнивании нулю “лишних" компонент решения с наибольшими номерами. Аналогичная процедура замыкания использовалась в статистической физике (где она называется "методом проектирования") и гидромеханике (см., например, [35]). Этот же подход (приравнивание нулю правых частей в системе (8)) мы применяем в задаче описания траекторий движения вихревых особых решений уравнения (1).

Разумеется, системы уравнений, получаемые в результате обрыва цепочек Гюгонио-Маслова, не могут точно определять движение особенности (или фронта особенности) на больших временных промежутках, так как обрыв цепочек означает насильственную локализацию задачи и описание эволюции особенности только по ее некоторой окрестности. Однако если такое описание применяется не для очень больших времен и если "оборванная" цепочка обладает некоторыми свойствами устойчивости, то использование таких “оборванных" цепочек кажется приемлемым ${ }^{10)}$.

Приведем соответствующие результаты, которые ранее были получены в несколько иной форме для $\omega=0$ в $[1,29]$ и для $\omega \neq 0$ в [9].

УТВЕРЖДЕНИЕ 1. Если у системы (1) существует решение вида (13), удовлетворяющее сформулированным выше условиям, то

а) $F=\sqrt{\tau}$

б) выполнень соотношения (5)-(7);

в) негладкая составляющая решения (функиии $\widetilde{\rho}=R \sqrt{S}$ и $\widetilde{u}=U \sqrt{S})$ восстанав-

\footnotetext{
${ }^{10)} \mathrm{C}$ другой стороны, например, в задаче о траектории "глаза тайфуна" получение данных, описывающих скорости $\mathbf{u}$ и геопотенциал $\eta$ в начальный момент времени, позволяющих хорошо поставить задачу Коши для системы (1), оказывается весьма проблематичным, ввиду возможных резких локальных колебаний скоростей и принципиальной невозможности организовать частую пространственную сетку измерения скорости ветра, давления и т.д. Траектория же движения "глаза тайфуна" достаточно достоверно определяется, например, со спутников, и можно пытаться предсказать будущую часть траектории тайфуна по ее известной части, решая задачу экстраполяции. Для этого, в свою очередь, можно использовать уравнения (9) для траекторий вихрей, полученные в результате интегрирования (оборванных) цепочек. Таким образом, по самой физической постановке локализация задачи представляется вполне разумной.
} 
ливается через $X(t), p(t), q(t)$ с помощью равенств

$$
\left(\begin{array}{c}
\tilde{u} \\
\tilde{\rho}
\end{array}\right)=A\left(\begin{array}{c}
\mathbf{T} \nabla S^{(2)}+O\left(|x-X(t)|^{2}\right) \\
2 c \rho_{0} S^{(2)}+O\left(|x-X(t)|^{3}\right)
\end{array}\right) \sqrt{S^{(2)}+O\left(|x-X(t)|^{3}\right)}
$$

əде

$$
S^{(2)}=\frac{1}{2} \exp \left(-\int_{0}^{t} q(t) d t\right)\left(x-X(t), \Pi(t) B \Pi^{*}(t)(x-X(t))\right), \quad B=\left(\begin{array}{cc}
b_{1} & 0 \\
0 & b_{2}
\end{array}\right),
$$

$b_{1}>0, \quad b_{2}>0, \quad b_{1} \neq b_{2}$,

$$
\Pi=\left(\begin{array}{cc}
\cos \theta & \sin \theta \\
-\sin \theta & \cos \theta
\end{array}\right)
$$

- матрица поворота на угол $\theta(t)=\theta_{0}+\int_{0}^{t} p(t) d t, \theta_{0}, A \neq 0, \quad c=\left(2 p-\omega_{0}\right) / \rho_{0} \neq 0$ - вещественнье константы, характеризующие начальную структуру вихревого решения;

г) для производных $\rho_{l j}, v_{l j}, w_{l j}$ и функиий $\omega_{0}(t), \quad V_{1}=v(X(t)), \quad V_{2}=w(X(t))$ дополнительно $\kappa(5)$ и (7) выполнены соотношения (8) с правыми частями

$$
\begin{gathered}
f^{r}=-\rho_{0}\left(3 v_{30}+3 w_{03}+w_{21}+v_{12}\right), \\
f_{20}^{v}=-3 \rho_{30}, \quad f_{11}^{v}=-2 \rho_{21}, \quad f_{02}^{v}=-\rho_{12} \\
f_{20}^{w}=-\rho_{21}, \quad f_{11}^{w}=-2 \rho_{12}, \quad f_{02}^{w}=-3 \rho_{03} .
\end{gathered}
$$

Условия (5)-(8) и представление (14) являются необходимыми и достаточными для того, чтобь функиия (2) удовлетворяла исходной системе уравнений с точностью до $O\left(|x-X(t)|^{3}\right)$.

Формула (14) достаточно проста, и ее анализ не составляет труда. Функция $\widetilde{u}$ описывает движение уединенного вихря по траектории Г поля скоростей $u(x, t)$. Этот факт не является удивительным - он хорошо известен в гидродинамике (см., например, [36]). Его наличие для решений вида (2) говорит о том, что такое решение не противоречит законам гидродинамики. Тем не менее для решений вида (2) он требует доказательства. Заметим, однако, что траектория Г может быть устроена довольно сложно. Любопытным является требование выполнения условий Коши-Римана (6). Эти условия не являются инвариантными для (любых) траекторий поля скоростей, и они, таким образом, описывают влияние существования вихря ${ }^{11)}$ на "гладкий фон" $u(x, t)$. Квадратичной форме $S^{(2)}$ соответствует “эллипс асимметрии” с полуосями, определяемыми числами $b_{1} \neq b_{2}$, и начальным углом $\theta_{0}$. Этот "эллипс асимметрии” при движении вдоль $X(t)$ вращается (в силу условий Коши-Римана) с мгновенной угловой скоростью $\dot{\theta}=-(1 / 2) \operatorname{rot}_{3} u(X(t), t)$. Заметим также, что “вихревая" (негладкая) часть решения

\footnotetext{
11)Условия Коши-Римана вместе с равенством $2 p-\omega_{0}=c \rho_{0}$ являются следствием сохранения вдоль траекторий векторного поля $\mathbf{u}$ потенциального вихря (инварианта Россби-Эртеля, см., например, $[12,37])\left(\mathbf{u}_{1 x_{2}}-\mathbf{u}_{2 x_{1}}+\omega\right) / \eta$.
} 
растет при удалении от $X(t)$ достаточно медленно - как $|x-X(t)|^{2}$, а функция $\widetilde{\rho}$ растет еше медленнее ${ }^{12)}$ - как $|x-X(t)|^{3}$. Это означает, что о негладкой составляющей решения (2) нельзя говорить как о "врашаюшемся твердом теле", поскольку в последнем случае касательные скорости растут линейно - как $|x-X(t)|$. Напомним, что мы рассматриваем только такие решения системы $(1)$, когда $\eta>0$. Следовательно, $\rho_{0}(t)>0$ и негладкая компонента плотности $\widetilde{\rho}$ имеет для всеx $t$ на траектории Г максимум, если $A c<0$, и минимум, если $A c>0$. Знаки у констант $A$ и $c$ никак не связаны друг с другом и, по всей видимости, выбор знака у $A$ (а следовательно, отбор решений с максимумом и минимумом геопотенциала на траектории) аналогичен выбору волны разрежения или сжатия в теории ударных волн [2-4]. Наконец, отметим, что описанная динамика вихря сохраняет свойство асимметрии. В первом приближении "эллипс асимметрии" преобразуется с помощью (локально) конформного преобразования: он поворачивается, растягивается и сжимается по всем направлениям одинаково, коэффициент растяжения определяется дивергенцией поля скоростей $\mathbf{u}(x, t)$ на траектории $X(t)$.

"Сжимаемость" $\eta$ в системе (1) играет важную роль как при выводе цепочки, так и при ее дальнейшем исследовании. Если вместо системы (1) рассмотреть двумерное уравнение Эйлера для несжимаемой жидкости (формально это означает, что в системе (1) уравнение непрерывности заменяется на уравнение $\operatorname{div} \mathbf{u}=0)$, то вектор-функции $X(t), V(t)$ не будут зацепляться с остальными производными от $u$ и $\rho$ и траектория $\Gamma$ может быть произвольно заданной ${ }^{13)}$. По-видимому, этот факт, вытекаюший из инвариантности уравнения Эйлера во всем пространстве по отношению к переходу к неинерциальной системе координат [38], можно объяснить тем обстоятельством, что краевые эффекты в несжимаемой среде влияют на траекторию особенности уже в нулевом приближении, в то время как в сжимаемой среде задачу о распространении особенности удается в некотором приближении “локализовать". Условие наличия “сжимаемости" при написании цепочки для точечных особенностей вполне согласуется с тем фактом, что для ударных волн условия Гюгонио пишутся для (сжимаемых) уравнений газодинамики. Наконец, отметим, что требование "структурной самоподобности” решения (2) приводит к тому, что асимптотически особенность (вихрь) двигается только по "гидродинамической моде" (вихрь “вморожен"в поле скоростей, см. (3)), не отдавая энергию в "акустические моды", наличие которых обусловлено сжимаемостью ${ }^{14)}$. Таким образом, имеет место еше один несколько парадоксальный факт: хотя включение в иепочку функиий, определяющих траекторию, возможмн благодаря наличию звуковых мод, однако “основная часть" вихря переносится по гидродинамической, а не по акустическим модам.

\footnotetext{
12) Такой медленный рост функций (14) является аргументом в пользу их применения для описания тайфунов - он соответствует наличию "глаза" у тайфуна.

13) Этот факт установил Г. В. Коваль.

14) Все три моды линеаризованной на гладком фоне системы (2) пересекаются в точках траектории $X(t)=(x: \nabla S(x, t)=0 \Longleftrightarrow S(x, t)=0)$.
} 


\section{4. АСИМПТОТИЧЕСКАЯ ИНТЕРПРЕТАЦИЯ ЗАМЫКАНИЯ ЦЕПОЧКИ}

Описанная в разделе 1 процедура замыкания означает обращение в нуль правых частей в уравнениях (8). Сформулированное ниже утверждение, вытекающее из анализа следующих за (8) (и приведенных в [9]) уравнений цепочки, проясняет ее смысл с точки зрения асимптотических разложений.

УТВЕРЖДЕНИЕ 2. Пусть коэффиииенты $\rho_{k}, v_{k}, w_{k}$ при $|k| \leqslant 2$ удовлетворяют уравнениям (3)-(5), (7), (8), $S, U, R$ определень с помощью (14). Тогда $u_{k} u$ $v_{k}, \quad k=\left(k_{1}, k_{2}\right), \quad k_{1}+k_{2}=3$, можнн выбрать так, что функиия (2) удовлетворяет исходной системе (1) с точностью до $O\left(|x-X(t)|^{3}\right)$ при любъх значениях тейлоровских коэффициентов для функиий $u=(v, w)$ с номерами $k=\left(k_{1}, k_{2}\right)$, $|k| \geqslant 4$, и для функций $U, R, S, \rho$ с номерами $|k| \geqslant 3$. В частности, полагая все указанные "стариие" коэффициенты равными нулю, получим приближенные решения (2) $\bmod O\left(|x-X(t)|^{3}\right)$ c $\mathbf{f}, U, R, S$, полиномиально (не выше 3 степени) зависящими от $(x-X(t))$.

Отсюда следует, что обрыв цепочки - ее замена на 17 уравнений $(5),(8)$, соответствует построению в некотором смысле асимптотического решения (по степеням $x-X(t))$ исходной системы уравнений ${ }^{15)}(2)$. Строгого доказательства того факта, что предложенное замыкание цепочки приводит к определению траектории особенности $X(t)$, мало отличающейся на некоторых временах от настоящей траектории вихря, пока нет ${ }^{16)}$. Однако, как видно из дальнейшего, интересующие нас решения замкнутой цепочки дол жны быть (и выбираются) устойчивыми. Появление свойства устойчивости позволяет надеяться доказать, что на некоторых временах правые части в системе (8) будут вносить достаточно малый вклад в решения, если допустить, что эти правые части не нулевые, но достаточно малые ${ }^{17)}$. С другой стороны, сфформулированное утверждение 1 позволяет полностью определить “старшую” часть негладкой составляюшей решения (2). Простые рассуждения, основанные на приведенном ниже представлении (15), (16) “оборванной” цепочки, показывают, что учет только слагаемых первого и нулевого порядков сильно меняет картину поведения решений системы, содержашей также и слагаемые второго порядка. Поэтому произведенньй обрыв цепочки является достаточно разумным: обрыв на предыдушем шаге (т.е. обрашение в нуль коэффициентов $v_{20}, w_{02}, v_{02}$ и т.д.) не позволяет правильно описать старшую негладкую часть, а учет следуюших уравнений приводит к многократному усложнению уравнений (обрыв цепочки на 3 шаге добавляет еше 12 уравнений, на 4 шаге - 27 и т.д.).

\footnotetext{
${ }^{15)}$ Грубо говоря, это означает, что замена функций $\mathbf{f}, U, R, S$ полиномами степени не выше 3 по $(x-X(t))$ оказывается разумной, если их коэффициенты (зависящие от $t$ ) выбраны подходящим образом.

16) Оценка для разности между решениями оборванной и необорванной цепочек, соответствующими ударным волнам уравнения Хопфа, получена недавно в [33].

17) Вероятно, соответствующие результаты могут быть получены не для любых, а для некоторых выделенных решений цепочки, в частности для обсуждаемых здесь решений с малой частотой $p-\omega_{0}$.
} 


\section{5. ИНТЕГРАЛЫ УКОРОЧЕННОЙ ЦЕПОЧКИ, НОВЫЕ ПЕРЕМЕННЫЕ И РЕДУКЦИЯ К УРАВНЕНИЮ ХИЛЛА}

Проведенная в [21] для случая постоянной силы Кориолиса редукция системы из 17 уравнений (5), (8) к уравнению Хилла (3) сильно упрошается после введения новых зависимых переменных. Более того, использование этих переменных позволяет далеко продвинуться в решении задачи о нахождении гладких траекторий (траекторий тайфунов, в частности) уже с учетом переменной силы Кориолиса (“ $\beta$-эффекта”). Опишем эти новые переменные, учитывая при этом, что одним из интегралов системы (5), (8) является значение $c$ потенциального вихря на траектории (см. утверждение 1). Введем комплексную координату $z=x_{1}+i x_{2}$ на плоскости $\left(x_{1}, x_{2}\right)$, комплексную регулярную составляющую скорости $\mathbf{v}=v(x, t)+i w(x, t)$, комплексную траекторию $\Gamma$ особенности (вихря) $X=X_{1}(t)+\left.i X_{2}(t) \equiv z\right|_{\Gamma}$, комплексную скорость особенности $V=V_{1}(t)+\left.i V_{2}(t) \equiv \mathbf{v}\right|_{\Gamma}$, производные $\partial / \partial z=\partial / \partial x_{1}-i \partial / \partial x_{2}, \partial / \partial \bar{z}=\partial / \partial x_{1}+i \partial / \partial x_{2}$ и следуюшие вешественные и комплексные переменные:

$$
\begin{gathered}
\nu=|c| \rho_{0}=\sigma\left(2 p-\omega_{0}\right), \quad Y=\left.\frac{1}{\nu^{\frac{3}{2}}|c|}\left(\frac{1}{2} \frac{\partial^{2} \mathbf{v}}{\partial z \partial \bar{z}}-\frac{\beta}{3}\right)\right|_{\Gamma}, \quad Z=\left.\frac{1}{\nu^{\frac{3}{2}}|c|}\left(\frac{1}{4} \frac{\partial^{2} \mathbf{v}}{\partial z^{2}}+\frac{\beta}{3}\right)\right|_{\Gamma}, \\
U=\left.\frac{1}{4 \nu^{\frac{3}{2}}|c|} \frac{\partial^{2} \mathbf{v}}{\partial \bar{z}^{2}}\right|_{\Gamma}, \quad W=\left.\frac{1}{\nu^{\frac{3}{2}}|c|}\left(\frac{c}{i} \frac{\partial \rho}{\partial \bar{z}}-\frac{1}{2} \frac{\partial^{2} \mathbf{v}}{\partial \bar{z} \partial z}+\frac{1}{2} \frac{\partial^{2} \overline{\mathbf{v}}}{\partial \bar{z}^{2}}+\beta\right)\right|_{\Gamma} \\
\lambda=\frac{1}{4}-\frac{2 r}{\nu^{2}}+\frac{|c|}{2} \operatorname{Re}\left((Z-\bar{Y}) W+\frac{3}{2} Y Z\right) .
\end{gathered}
$$

Во введенных переменных "оборванная" цепочка принимает вид

$$
\begin{gathered}
\dot{V}-i \omega_{0} V+i \sigma(\nu)^{\frac{3}{2}}(Y+W-2 \bar{Z})=0, \quad \dot{Y}=i\left(p-\omega_{0}\right) Y-\frac{i \beta\left(2 p+\omega_{0}\right)}{3|c| \nu^{\frac{3}{2}}}, \\
\dot{Z}=i\left(3 p-\omega_{0}\right) Z+\frac{i \omega_{0} \beta}{3|c| \nu^{\frac{3}{2}}}, \quad \dot{U}=i\left(p+\omega_{0}\right) U, \quad \dot{W}=-i p W \\
\dot{\lambda}=-\frac{\beta}{\nu^{\frac{3}{2}}} \operatorname{Im}\left(\frac{2 p}{3} W-\frac{\omega_{0}}{2} Y+\frac{2 p+\omega_{0}}{2} Z\right), \quad \dot{\omega}_{0}=\beta \operatorname{Im} V, \\
\frac{1}{2} \frac{d}{d t}\left(\frac{1}{\nu} \frac{d \nu}{d t}\right)-\frac{1}{4}\left(\frac{1}{\nu} \frac{d \nu}{d t}\right)^{2}+c^{2} Q \nu^{2}-b^{2}=0 \Longleftrightarrow \frac{d^{2} \sqrt{\nu}}{d \Phi^{2}}+Q \sqrt{\nu}=\frac{b^{2}}{(\sqrt{\nu})^{3}} .
\end{gathered}
$$

Здесь

$$
\begin{gathered}
\Phi=\sigma \int_{0}^{t} \nu d t, \quad b^{2}=\frac{\omega_{0}^{2}+2 \beta \operatorname{Re} V}{4}, \\
\sigma=\operatorname{sign} c, \quad Q=\lambda+c \operatorname{Re}\left(Z W-\bar{Y} W+\frac{3}{2} Y Z\right), \quad p=\frac{\omega_{0}+\sigma \nu^{3}}{2} .
\end{gathered}
$$

Преобразование уравнений (5), (8) (с нулевыми правыми частями) к виду (15), (16) оказывается вполне элементарным, если известен окончательный результат. Это преобразование состоит в умножении уравнений (8) на подходящие коэффициенты, их сложении и последуюшем "убивании" диагонального слагаемого $3 q$. Но именно это преобразование [9] (индуцированное обнаруженной в [21] редукцией) представляет собой наше 
основное наблюдение, которое приводит к выводу уравнений Хилла (3), "почти физического маятника" (9), указанных ниже "резонансных" соотношений между частотами и т.д.

Из представления (15), (16) “оборванной” цепочки видно, что ее решения имеют осиилляиионныц характер с физически разумными частотами $p-\omega_{0}, 3 p-\omega_{0}, p, \omega_{0}$ (напомним, что $p(t)$ - угловая скорость врашения “эллипса асимметрии” вихря). Кроме того, это представление при $\beta=0$ позволяет немедленно найти в дополнение к интегралу (см. утверждение 1) с другие интегралы “оборванной” цепочки: $|Y|,|Z|,|U|,|W|, \lambda$. Редукция к уравнению Хилла (3) вытекает из следующих соображений: потенциал в нем совпадает с потенциалом $Q$ в уравнении $(16)$ для $\sqrt{\nu}$, которое называется уравнением Ермакова и точно сводится к семейству уравнений (3) (см. [39], формула (13)). Этот факт позволяет достаточно явно выразить через решения уравнения Хилла все решения “оборванной" цепочки, по крайней мере грубо описать их свойства, и, в частности, связать свойства траекторий с зонной теорией устойчивости. По физическому смыслу на рассматриваемых временных промежутках геопотенциал $\eta$, и следовательно $\rho_{0}$, должны быть ограничены сверху и снизу положительными константами (которые в задаче о тайфунах к тому же не очень сильно отличаются друг от друга). Используя известные свойства устойчивости уравнений Хилла и явные формулы, выражающие функции $\rho_{0}(t)$ и $X(t)$ через решения уравнения Хилла, можно прийти к следуюшим важным выводам.

1. Частота Кориолиса $\omega$ должна быть отлична от нуля.

2. Параметры в уравнении Хилла (т.е. константы интегрирования “оборванной” цепочки) должны быть такими, чтобы оно было устойчивым, или по крайней мере соответствовали достаточно узкой зоне неустойчивости (с маленькими инкрементами и декрементами неустойчивости). Получается также, что в устойчивом случае функция $\rho_{0}(t)$ оказывается квазипериодической с двумя частотами, причем одна из них равна $\omega_{0}$.

\section{6. РЕЗОНАНСНЫЕ (КРИТИЧЕСКИЕ) РЕЖИМЫ}

Еще одно физически разумное предположение о достаточной гладкости траекторий Г и вполне элементарное исследование уравнения для скорости приводят к требованию малости осциллируюшей части потенциала в уравнении Хилла и малости одной из частот $p-\omega_{0}, 3 p-\omega_{0}, p$ системы (15). Простые вычисления показывают, что такие ситуации возможны в случае, когда параметр $\lambda$ лежит в окрестности чисел $1 / 4$ и $9 / 4$, иначе говоря, в окрестности первой и третьей лакун спектра уравнения Хилла. Подмногообразия решений “оборванной” цепочки, соответствуюшие таким ситуациям, естественно назвать резонансным.м или критическими, поскольку первые два случая дают соотношения между частотой Кориолиса и угловой скоростью врашения “эллипса асимметрии" вихря:

$$
\mathrm{I}: \quad p \approx \omega_{0}, \quad \text { II }: \quad 3 p \approx \omega_{0}
$$

Представляется весьма правдоподобным, что именно эти режимы соответствуют движениям мезомасштабных вихрей в атмосфере и отбирают среди решений “оборванной” цепочки инвариантные подмногообразия, представляюшие собой нечто вроде $а т m p a \kappa-$ торов. Любопытным и, вероятно, опять не случайным является тот факт, что резонанс- 
ные режимы исследуются достаточно просто. В частности, эти исследования проясняют роль силь Кориолиса в динамике мезомасштабных вихрей.

Заметим, что система (15), (16) (или ее соответствующие эквивалентные формы) инвариантна по отношению к замене переменных: $p \rightarrow-p, \omega_{0} \rightarrow-\omega_{0}, c \rightarrow-c, V \rightarrow \bar{V}$, $Y_{j} \rightarrow \bar{Y}_{j}$ (черта означает комплексное сопряжение). Поэтому без уменьшения общности будем предполагать, что $\omega_{0} \geqslant 0$ (т.е. что наши рассмотрения проводятся в северном полушарии; переход в южное полушарие осушествляется с помощью соответствуюшей замены).

\section{7. УРАВНЕНИЕ ТИПА ФИЗИЧЕСКОГО МАЯТНИКА ДЛЯ ГЛАДКИХ ТРАЕКТОРИЙ РЕЖИМА I. ОТБОР “СУЩЕСТВЕННЫХ" ПАРАМЕТРОВ}

Обсудим здесь решения, соответствуюшие только режиму І. Свойства решений, соответствуюших режиму II, оказываются весьма близкими [9]. Нетрудно показать, что для режима I $c>0$ и $\sigma=1$. Сначала предположим, что $\beta=0$. Если осциллируюшая часть потенциала $Q$ в (16) (или в (3)) достаточно мала и, в частности, равна нулю и если частота Кориолиса отлична от нуля, тогда легко видеть, что у уравнения Ермакова (16) для $\sqrt{\nu}$ есть “потенциальная яма". Это позволяет построить точные решения [9] с постоянными значениями $\rho_{0}$, которым соответствуют совсем простые формулы для траекторий. Например, выбирая $Z=W=0, p=$ const, $\omega=\omega_{0}=$ const, $\lambda \approx 1 / 4$, получаем точную формулу (4).

В разделе 1 мы уже отмечали еще один неожиданный, но, видимо, не случайный факт, состоящий в том, что осредненные уравнения для резонансных режимов I и II оказываются интегрируемыми в квадратурах и близки к уравнениям "почти физического маятника" (8). Еще один важный аспект, касающийся вывода системы (8), состоит в следуюшем. Сама процедура осреднения (хотя и проведенная на физическом уровне строгости, но полностью подтвержденная расчетами на компютере) выделяет из 17 констант интегрирования "оборванной" цепочки "сушественные" константы $X_{1}^{0}, X_{2}^{0}$, $\Omega, R, \psi(0)$, которые сильно влияют на "грубое" поведение траекторий. Эти константы полностью определяются с помощью физически разумных начальных характеристик вихря - положения его центра $\left(X_{1}^{0}, X_{2}^{0}\right)=\left(X_{1}(0), X_{2}(0)\right)$, поступательной скорости $V_{1}(0), V_{2}(0)$, угловой скорости врашения вихря $p(0)$ :

$$
\begin{gathered}
\omega_{0}(0)=\omega\left(X_{2}(0)\right) \approx \widetilde{\omega}+\beta X_{2}(0), \quad \Omega=\frac{9 \omega_{0}(0)}{4\left(2-\frac{p(0)}{\omega_{0}(0)}\right)^{2}}, \\
R=\frac{|V(0)|}{K\left(\omega_{0}(0), \Omega\right)}, \quad \psi(0)=\arg V(0)
\end{gathered}
$$

Один из основных результатов работы и состоит в “правильном" выделении (фильтрачии) из 17 параметров (начальных данньх, задающих решения "оборванной” чепочки) таких, которые приводят к гладким траекториям. Влияние других параметров на поведение решений системы (15), (16) мы обсудим немного позднее. 


\section{8. КЛАССИФИКАЦИЯ ВОЗМОЖНЫХ ТРАЕКТОРИЙ МЕЗОМАСШТАБНЫХ ВИХРЕЙ РЕЖИМА І}

Перейдем к классификации траекторий вихрей. На плоскости $\left(\psi, \omega_{0}\right)$ точки $\left(\psi, \omega_{0}\right)=$ $(2 \pi l, 4 \Omega / 9)$ являются центрами, точки $\left(\psi, \omega_{0}\right)=(\pi(2 l+1), 4 \Omega / 9)$ - седловыми (гиперболическими) точками. Если в (12) const $=R \beta / \Omega^{3 / 2}$, то это равенство определяет сепаратрисы, разделяюшие фазовую "полосу" на области финитного и инфинитного движений. Каждый из этих типов движения определяет различные траектории $x=X(t)$ на $\beta$-плоскости.

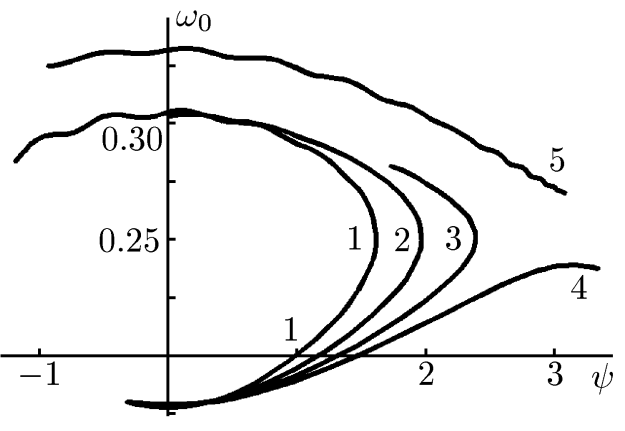

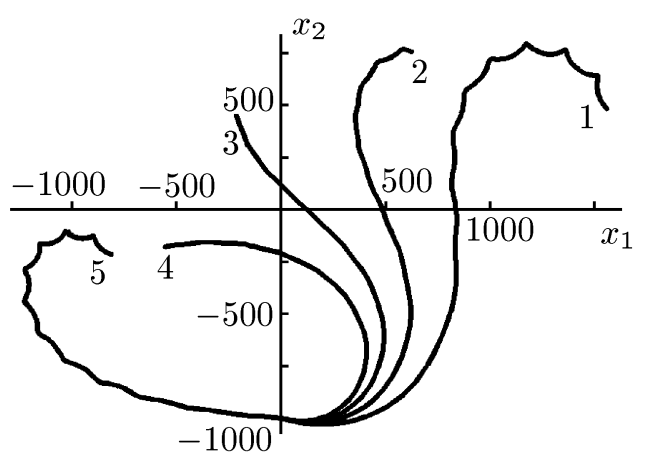

6

Рис. 5

Мы хотим сопоставить траекториям $x=X(t)$, полученным с помощью приближенного интегрирования, траектории реальных тайфунов. Известно, что, как правило, тайфуны в северном полушарии на протяжении почти всего своего существования двигаются с юга на север и лишь непродолжительное время после возникновения и перед исчезновением могут двигаться с севера на юг (некоторые отклонения от такого движения иногда возникают в средней части траекторий, что, по-видимому, соответствует переходу с одного режима на другой, вызванному неучтенным в (1) механизмом "подкачки" энергии). Таким образом, сушествует некоторый запрет на продолжительное движение тайфунов с севера на юг в северном полушарии. "Оборванная” цепочка (5), (8) такой запрет не содержит. С большой вероятностью не содержит его и исходная система уравнений мелкой воды (1), и для получения соответствующих ограничений следует рассматривать более сложные модели. Поэтому мы априори будем рассматривать только такие временные промежутки и такие решения как полной “оборванной” цепочки (5), (8), так и укороченной (осредненной) системы (9), которые описывают именно указанные траектории (или их части) $\Gamma=(x=X(t))$. Это, в частности, означает, что на фазовой плоскости $\left(\omega_{0}, \psi\right)$ нас будут интересовать лишь участки кривых $(10)$ в прямоугольнике $0<\delta_{1}<\omega_{0}<\Omega-\delta_{1},-\delta_{2}<\psi<\pi+\delta_{2}$, где $\delta_{1}$ и $\delta_{2}-$ некоторые малые положительные константы, и времена, если и превышаюшие, то незначительно, полупериод системы (9). 
Тогда мы имеем следующую топологическую классификацию движений в Этом прямоугольнике и соответствующих им траекторий на плоскости $\left(x_{1}, x_{2}\right)$ (рис. $5 \mathrm{a}-$ фазовые портреты, рис. $5 б$ - траектории):

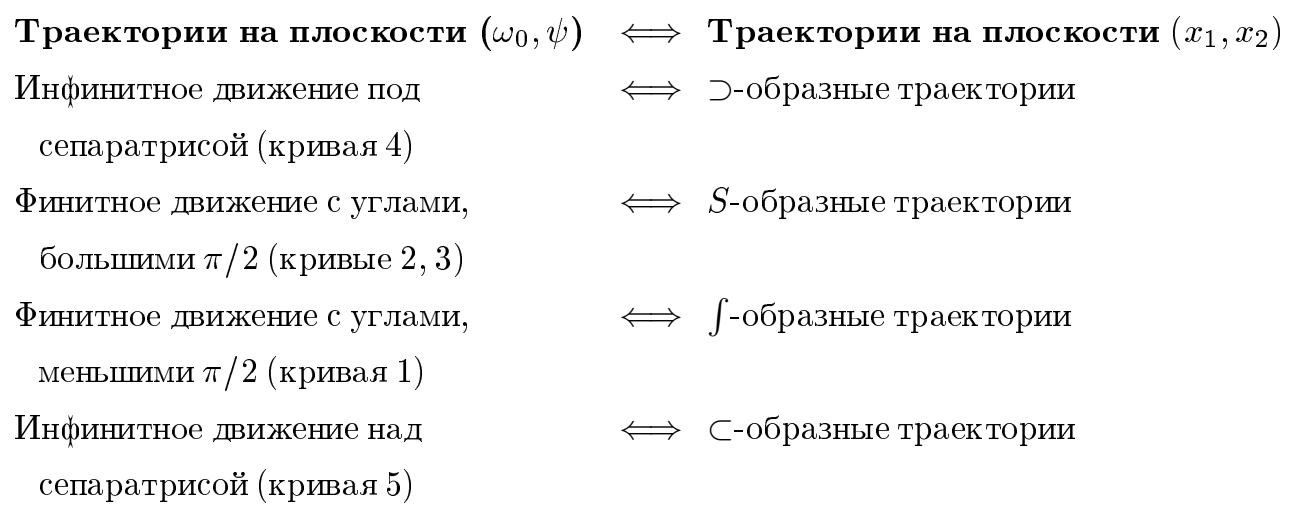

Мы сразу приводим траектории и фазовые портреты полной “оборванной" иепочки. Мелкие “дрожания" траекторий вызваны специально внесенными возмущениями критических режимов, их появление мы обсудим в следующем разделе.

Топологически случаи $S$ - и $\int$-образных траекторий эквивалентны, нам, однако, с точки зрения приложений разумно рассматривать их раздельно. Сепаратрисе и близким к ней кривым соответствует “вытягивание" траекторий вдоль “сепаратрисной” параллели, соответствуюшей удвоенному значению частоты Кориолиса 4 $/ 9$. Приближение к центру также соответствует движению вдоль этой же параллели.

\section{9. ВЛИЯНИЕ НА ТРАЕКТОРИЮ ВИХРЯ ЗНАЧЕНИЯ ПОТЕНЦИАЛЬНОГО ВИХРЯ $c$ И "НЕСУШЕСТВЕННЫХ" ПАРАМЕТРОВ. РОЛЬ УРАВНЕНИЯ ХИЛЛА}

Если не предполагать, что $c V^{2} \gg \omega_{0}$, то $R$ становится функцией $\omega_{0}$ (и параметров $\Omega$, $\left.R_{0}\right)$ :

$$
R^{2}=\left(R_{0}\right)^{2}-\frac{2}{|c|}\left(\frac{1}{27} \frac{4-3 \sqrt{\frac{\omega_{0}}{\Omega}}}{\sqrt{\frac{\omega_{0}}{\Omega}}-1}-\frac{1}{27} \frac{4-3 \sqrt{\frac{\omega_{0}(0)}{\Omega}}}{\sqrt{\frac{\omega_{0}(0)}{\Omega}}-1}\right)
$$

второе уравнение из (9) заменяется на уравнение

$$
\dot{\psi}=N\left(\omega_{0}, \Omega\right)-\frac{\beta G\left(\omega_{0}, \Omega\right)}{c R} \cos \psi
$$

где

$$
G\left(\omega_{0}, \Omega\right)=\frac{5-3 \sqrt{\frac{\omega_{0}}{\Omega}}}{3^{\frac{5}{2}} \sqrt{\omega}_{0}\left(1-\sqrt{\frac{\omega_{0}}{\Omega}}\right)^{\frac{3}{2}}}
$$


а вместо (12) мы имеем

$$
\begin{aligned}
\cos \psi= & \exp \left(\int_{\omega_{0}(0)}^{\omega_{0}} \frac{G(\xi, \Omega) d \xi}{c\left(R\left(\xi, \Omega, R_{0}\right)\right)^{2} K(\xi, \Omega)}\right)\{\cos \psi(0)- \\
& \left.-\int_{\omega_{0}(0)}^{\omega_{0}} \exp \left(-\int_{\omega_{0}(0)}^{\zeta} \frac{G(\xi, \Omega) d \xi}{c\left(R\left(\xi, \Omega, R_{0}\right)\right)^{2} K(\xi, \Omega)}\right) \frac{N(\zeta, \Omega) d \zeta}{\beta R\left(\zeta, \Omega, R_{0}\right) K(\zeta, \Omega)}\right\} .
\end{aligned}
$$

Анализ формулы (19) показывает, что при движении по траектории $X(t)$ с юга на север слагаемое $i \beta(2 p+\omega) /\left(c \nu^{3 / 2}\right)$ в системе $(9),(18)$ играет роль трения - скорость $V$ убывает экспоненциально, причем тем быстрее, чем меньше $c$. Таким образом, чем меньше значение потенциального вихря на траектории, тем больше "трение".

Мы уже отмечали, что компьютерное моделирование для полной “оборванной ” цепочки подтверждает правильность асимптотического вывода уравнений (9), а также и уравнения (18). Вместе с тем оно показывает, что для устойчивого выхода на режим следует соответствующим образом подбирать и начальные условия для переменных $Y, W$, $Z, \lambda$ и т.д., включив в них поправки $O(\beta)$. В противном случае у решений “оборванной" цепочки появляются быстрые осцилляции, а у траекторий - сначала “дрожания", а затем большие петли. Появление "дрожаний" и петель объясняется влиянием начальных возмушений на гладкие траектории. Грубый анализ системы ${ }^{18)}(15),(16)$ показывает, во-первых, наличие дополнительных резонансных частот у линеаризованной системы (на решениях режима I) и, во-вторых, что возмущения гладких траекторий разделяются на две группы. Возмущения из первой группы приводят лиш к изменению “существенных" параметров и сохраняют свойство гладкости (например, изменяют лишь период движения на плоскости $\left.\left(\omega_{0}, \psi\right)\right)$. Возмушения второй группы приводят к появлениям быстрых осцилляций и возникают, если, например, в начальный момент времени выбрать $W, Z$ отличными от нуля и в равенства (17) внести малые добавки. Именно такие возмушения и приведут к появлению быстрых осциллящий у этих функций, а следовательно, и у потенциала уравнения Хилла (3) в окрестности точки $\lambda=1 / 4$, являющейся следом первой зоны неустойчивости уравнения Хилла. Эти осцилляции "раскрьвают" зону неустойчивости и становятся тем больше, чем больше параметр $c$. Отклонение начального значения $\nu$ от гладкого режима приводит к осциллирующей добавке с быстрой фазой, находяшейся в резонансе с “собственными” колебаниями скорости (с частотой $\omega_{0}$, см. первое уравнение из системы $\left.(15)\right)$, и эта добавка тем больше, чем больше c. Поэтому такие отклонения начальных данных от данных, соответствующих гладким траекториям, приводят к отмеченному выше появлению “дрожаний” и бросков у траектории, а затем по мере их увеличения - к появлению у траекторий больших петель и в конце концов к быстрому хаотическому движению, причем “дрожание” и петли становятся тем больше, чем больше константа $c$. По всей видимости, включение в исходную

\footnotetext{
18) Если продолжить проводить аналогию с маятником, то можно сказать, что движение в силу полной системы $(15),(16)$ похоже на ситуацию, когда нить маятника упруго изменяет длину, точка подвеса прикреплена к пружинам, а сам маятник к тому же является не точкой, а вращающимся твердым телом.
} 
модель (1) вязких слагаемых, а также третьей переменной должно привести к быстрому затуханию такого сорта движений.

Таким образом, с учетом сказанного в начале этого раздела должен существовать некоторьй оптимальный интервал для значений потенциального вихря на траектории $(x=X(t))$ : малые $c$ означают наличие большого "трения", а большие $c$ приводят к петлям и хаотическому движению. Наконец, заметим, что в отличие от указанных физически разумных, измеряемых и наблюдаемых переменных $X, \omega, V$ и, возможно, $p, \rho_{0}$, $c$, оставшиеся переменные $Z, W$ и т.д., вероятно, являются плохо наблюдаемыми, и их вообще следует считать случайными. Тогда описанные гладкие траектории $(x=X(t))$ будут определять “середину коридоров”, в которых лежат возможные траектории центров вихрей.

Несмотря на существование указанных типов неустойчивостей, при правильном выделении начальных данных для полной “оборванной” цепочки гладкие траектории легко обнаруживаются на компьютере, причем в той области “сушественных "параметров (и этот факт представляется нам весьма знаменательным с точки зрения приложения цепочек Гюгонио-Маслова к задаче движения тайфунов), которая соответствует параметрам реальных тайфунов. С другой стороны, тайфуны - не столь частое явление, и для их существования (как и для образования) нужно выполнение целого ряда жестких требований, что, по-видимому, и может отражаться в наличии разного рода неустойчивостей в грубой модели.

Иллюстрацию проведенным рассуж дениям дают рис. 6а-6е, на которых изображены траектория и различные компоненты решения полной исходной системы с ненулевыми (но небольшими) “осциллирующими” компонентами $Z, W$. Заметим, что геопотециал на траектории изменяется достаточно мало.

На рис. 7 изображены траектории, отвечающие различным значениям параметра $c$, и соответствуюшие им фазовые портреты (кривая 1 соответствует $c=1$, кривая $2-c=$ 100 и кривая $3-c=0.01)$. Видно, что малые $c$ приводят к замедлению движения, а большие $c-$ к появлению бросков и петель. Всюду взято время 240 часов.

Наконец, на рис. 8 показана траектория (временной интервал тот же) с начальными данными, рассогласованными с начальными данными из режима I. Траектория принимает хаотический характер со множеством петель.

\section{0. СРАВНЕНИЕ ТЕОРЕТИЧЕСКИХ ТРАЕКТОРИЙ И ТРАЕКТОРИЙ РЕАЛЬНЫХ ТАЙФУНОВ}

Это сравнение [9] указывает на следующие факты: имеется качественное и “топологическое" совпадение траекторий режима I и траекторий реальных тайфунов и соответсвуюших им фазовых портретов на плоскости $\left(\omega_{0}, \psi\right)$ (удвоенная частота Кориолиса, угол направления поступательной скорости). При этом наиболее интересным является повторение зигзагов у ряда тайфунов. Оказывается также, что параметры траекторий реальных тайфунов (местоположение, скорость, угловая скорость врашения "эллипса асимметрии") таковы, что соответствующие фазовые портреты близки к сепаратрисным режимам. 

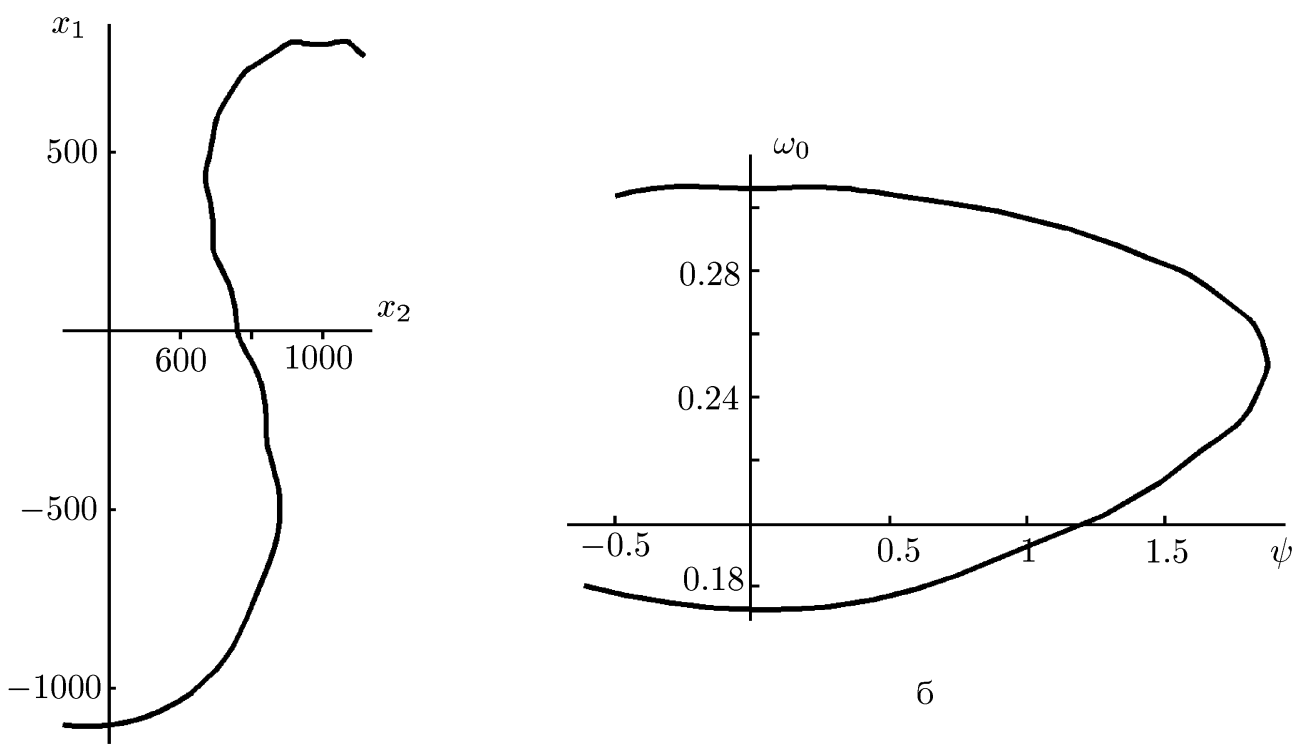

б
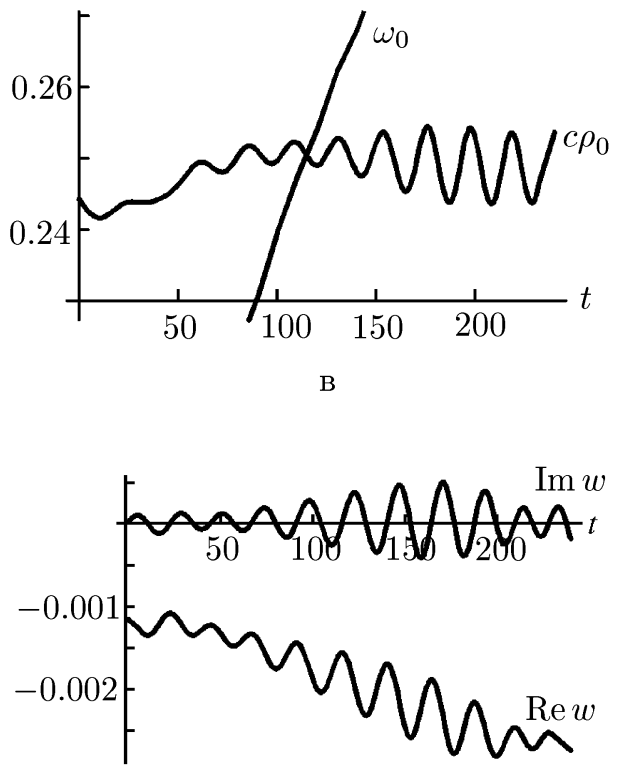

Д

Рис. 6

С другой стороны, имеется существенный недостаток в использовании уравнений (15), (16). Из анализа движения реальных тайфунов легко выводится следующий факт: по мере движения с юга на север увеличивается и максимальная угловая скорость вра- 

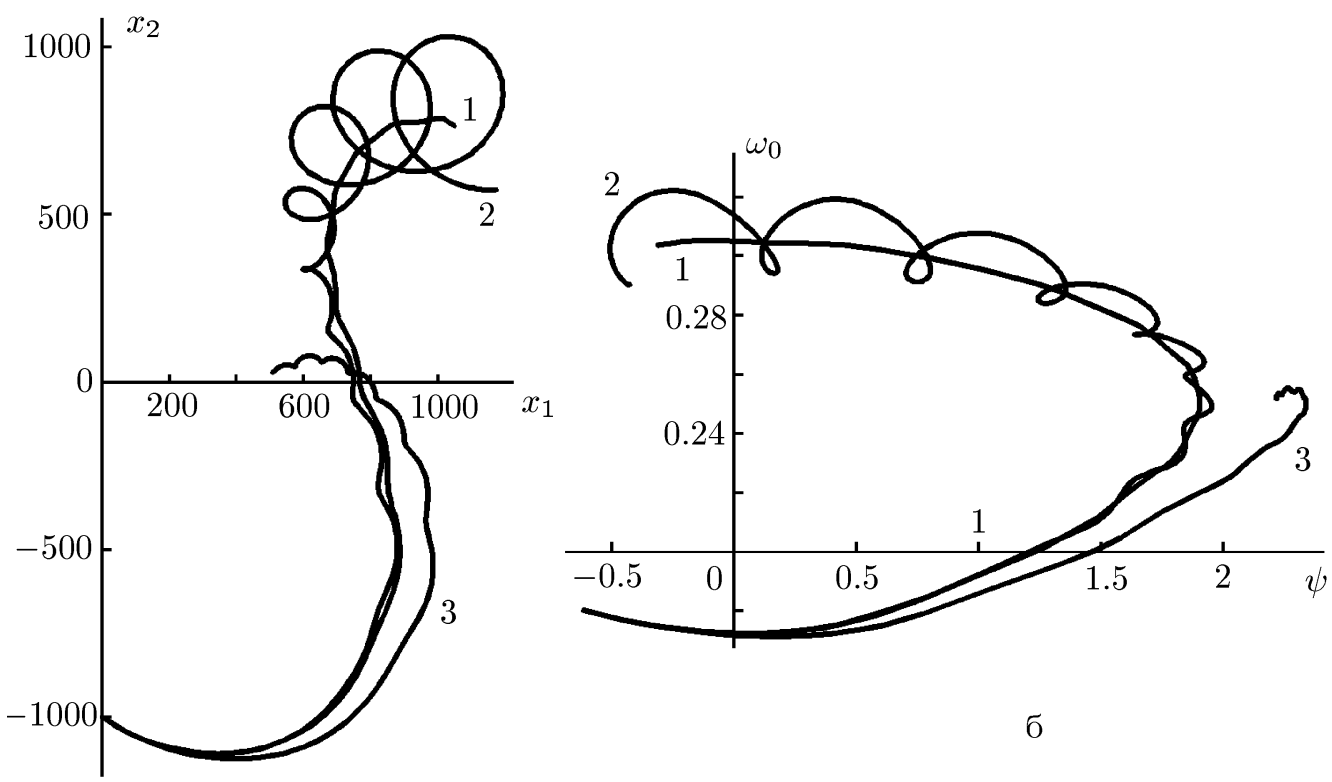

a

Рис. 7

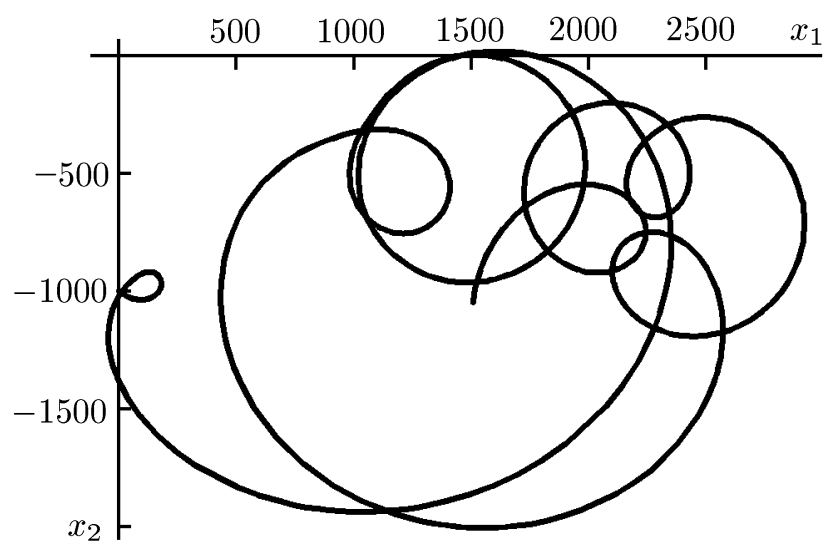

Рис. 8

шения, и поступательная скорость движения их центра. Если предположить, что максимальная угловая скорость врашения тем больше, чем больше угловая скорость вращения "эллипса асимметрии", то факт ее роста содержится в первой из формул (11). Поступательная же скорость $V$ в соответствии с формулой (10) убывает. Этот факт и приводит к не очень хорошему количественному совпадению реальных и теоретических траекторий: реальный тайфун проходит участки “теоретических траекторий” за сушественно более короткий промежуток времени (“убегает” от “теоретического” тайфуна). 
Конечно, уменьшение поступательной скорости можно пытаться объяснить за счет обрыва полной исходной цепочки, однако более разумное объяснение, по-видимому, содержится в факте чересчур грубого выбора исходной модели. Действительно, если представить, что мезомасштабный вихрь аналогичен движущемуся вращающемуся “твердому телу", то его энергия складьвается из энергий вращательного и поступательного движений, каждая из которых, разумеется, увеличивается с увеличением соответствующей скорости. Поэтому для консервативной модели, каковой является система уравнений мелкой воды, одновременное увеличение поступательной и врашательной скоростей невозможно: нужна "подкачка" энергии. Этот факт вполне согласуется с натурными наблюдениями и мнением специалистов: в образовании и движении тайфунов важную роль играют температурные (тепловые) эффекты и энергетическая "подкачка" океанической поверхностью. С точки зрения исходной модели это означает необходимость добавления к (1) уравнений, учитывающих внешние притоки тепла (см. п. 1.2 в статье [12]). Метод вывода соответствующей цепочки в этом случае несколько усложняется, но принципиальных трудностей в его реализации не видно, хотя, разумеется, интегрируемость укороченных систем может потеряться. Мы постараемся разобраться с этой ситуацией в последуюших публикациях. Вероятно, включение тепловых эффектов должно привести в уравнениях типа (15), (16) к конкуренции энергетической “подкачки" и потенциального вихря (инварианта Россби) $c$, создающего "трение": как только энергия перестает поступать, поступательная скорость движения может начать резко падать (см. формулу (19) и рис. 7), после чего и сам вихрь начинает разваливаться. Все эти соображения, конечно, являются не более чем гипотезами и требуют дальнейших, вероятно не простых, исследований.

\section{1. ЗАКЛЮЧИТЕЛЬНЫЕ ЗАМЕЧАНИЯ}

Несмотря на приведенную критику модели, задаваемой уравнениями (1) и (14), (15), нам кажется, что эта модель и полученные из нее "оборванные" цепочки дают некоторое описание движений реальных мезомасштабных вихрей и могут служить базой для дальнейших исследований более сложных моделей. В пользу этого соображения можно привести следующие аргументы.

Во-первых, совершенно неожиданно в результате реализации нетривиальных математических соображений и весьма объемных аналитических вычислений появляется много интересных математических объектов и фактов, порождаюших некоторые физически разумные следствия: условия Коши-Римана на выделенной траектории, уравнение Хилла, разумные резонансные соотношения между частотами и соответствующие им критические режимы, роль силь Кориолиса в их появлении, интегрируемость “осредненных" уравнений, описьвающих критические режимь, и появление фазовых портретов, схожих с фазовыми портретами физического маятника, простота окончательных математических формул ${ }^{19)}$ и т. д.

\footnotetext{
19) Видимо, сюда же можно отнести несколько загадочный факт одновременного совпадения резонансной частоты Кориолиса $\omega_{0}=p$ со значениями $\omega_{0}$, для которых функция $\rho\left(\omega_{0}\right)$ имеет максимум, а спектральный параметр $\lambda$ в уравнении Хилла является следом первой зоны неустойчивости.
} 
Во-вторых, прочедура въделения гладких траекторий и описание их с помощью методов осреднения приводит к выделению из 17 параметров пяти (возможсно, шести) физически разумных наблюдаемых “существенных" параметров траекторий вихрей, лежащих в диапазоне реальных параметров - скоростей, времени, коэффициентов $\beta$ и т.д. Именно для этих параметров траектории “оборванной” чепочки оказьваются достаточно гладкими, причем они совершают больиие ("глобальные") повороты и зигзаги именно такой формы, как у реальных тайфунов.

Исследования, предпринятые в этой работе, приводят к весьма на наш взгляд любопытным и, вероятно, нетривиальным вопросам как для специалистов в области математической физики, так и для специалистов в области физики атмосферы. K их числу относятся, в частности, следующие вопросы:

1) о выводе и анализе цепочки Гюгонио-Маслова для модели, учитывающей энергетическую "подкачку" вихря;

2) о гамильтоновости полной и "оборванной” цепочек Гюгонио-Маслова, о выявлении глубинных (вероятно, групповых) причин появления эффектов типа интегрируемости, смысле найденных интегралов, соответствуюших им переменных и т.д.;

3) о выводе следующих уравнений цепочек, соответствующих “сингулярной” и "регулярной" частям решения (что может привести к дополнительным соотношениям на $\left.u_{20}, w_{20}, u_{11}\right)$, и об оценке вклада в траекторию вихря отброшенных уравнений цепочки (вероятно, эта оценка может быть существенно более содержательной именно для траекторий выделенных критических режимов);

4) об устойчивости построенных вихревых решений как с точки зрения исходной, так и более общих моделей;

5) об аккуратном обосновании вывода укороченных систем и "осреднения";

6) об учете сферичности Земли при выводе и анализе цепочек и ее влиянии на траектории рассмотренных вихрей;

7) о включении в модель вязких слагаемых, которые должны привести к появлению “профиля" вихря, и связи с параметрами, его определяюшими;

8) о выводе цепочек Гюгонио-Маслова для вихревых образований в 3-мерных ситуациях, для взаимодействующих вихрей и т.д.

Вероятно, в исследовании перечисленных вопросов могут сыграть важную роль соображения и подходы, изложенные, в частности, в [12, 37, 40-48].

Наконец, еще раз повторим, что как рассматриваемая в этой работе модель, так и применяемые асимптотические разложения являются весьма грубыми приближениями. Нам представляется очень интересным их дальнейшее уточнение и, возможно, соединение с имеющимися теориями (например, [18, 43-47]), описывающими зарождение и гибель вихрей, их устойчивость, 3-мерную структуру (а значит, влияние спиральности), взаимодействие и т.д.

Благодарности. Я выражаю свою искреннюю благодарность Л.А. Багирову, В. В. Булатову, Ю. В. Владимирову, В. Г. Данилову, В. Ф. Должанскому, В. А. Гордину, Г. В. Ковалю, А.Г. Куликовскому, Е. Л. Коротяеву, В. П. Мясникову, А. И. Нейштадту, 
А. И. Шафаревичу и особенно В.П. Маслову за многочисленные стимулирующие дискуссии и ценные советы. Эта работа была выполнена при частичной поддержке гранта РФФИ № 99-01-00415.

\section{Список литературы}

[1] В. П. Маслов. УМН. 1980. Т. 35. № 2. С. 252-253.

[2] Г. Г. Черный. Газовая динамика. М.: Наука, 1986.

[3] А.Г. Куликовский, Е. И. Свешникова. Нелинейные волны в упругих средах. М.: Московский лицей, 1998.

[4] Джк. Б. Уизем. Линейные и нелинейные волны. М.: Мир, 1977.

[5] F. V. Shugaev, L. S. Shtemenko. Propagation and Reflection of Shock Waves. Singapore: World Sci., 1998.

[6] В. П. Маслов. О распространении ударной волны в изоэнтропическом невязком газе. В сб.: Итоги науки и техники. Соврем. проблемы математики. Т. 8. Ред. Р. В. Гамкрелидзе. М.: ВИНИТИ, 1977. С. 199-271.

[7] В. П. Маслов, Г. А. Омельянов. Сиб. матем. журнал. 1983. Т. 24. № 5. С. 172-182.

[8] М. А. Гринфельд. Прикладная математика и механика. 1978. Т. 42. № 5. С. 883-898.

[9] S. Yu. Dobrokhotov. Russ. J. Math. Phys. 1999. V. 6. № 2. P. 137-173; № 3. P. 282-313.

[10] В. Г. Данилов, В. П. Маслов, В. М. Шелкович. ТМФ. 1998. Т. 114. № 1. С. 3-55.

[11] А. М. Обухов. Изв. АН СССР. Сер. геогр. 1949. Т. 13. № 4. С. 281-306.

[12] Ф. В. Должанский, В. А. Крымов, Д. Ю. Манин. УФН. 1990. Т. 160. № 7. С. 1-47.

[13] Г. С. Голицын. Введение в физику планетарных атмосфер. Л.: Гидрометеоиздат, 1973.

[14] В. А. Гордин. Математические задачи гидродинамического прогноза погоды: аналитические аспекты. Л.: Гидрометеоиздат, 1987.

[15] J. Pedlosky. Geophysical Fluid Dynamics. Berlin: Springer, 1982.

[16] А. П. Хаин, Г. Г. Сутырин. Тропические циклоны и их взаимодействие с океаном. Л.: Гидрометеоиздат, 1983.

[17] Л. Д. Ландау, Е. М. Лифииц. Гидродинамика. М.: Наука, 1986.

[18] G. M. Reznik. J. Fluid Mech. 1992. V. 240. P. 405-432.

[19] В. М. Гряник. Океанология. 1988. Т. 26. С. 174.

[20] В. В. Булатов, Ю. В. Владимиров, В.Г. Данилов, С. Ю. Доброхотов. ДАН. 1994. T. 338. № 1. C. 102-105.

[21] С. Ю. Доброхотов. ТМФ. 1997. Т. 112. № 1. С. 47-66.

[22] С. Ю. Доброхотов. Цепочки Гюгонио-Маслова уравнения Хилла и физического маятника для траекторий мезомасштабных вихрей на "мелкой воде". Препринт № 663. М.: Инст. пробл. механики РАН, 2000.

[23] В. П. Маслов. Теория возмущений и асимптотические методы. М.: МГУ, 1965.

[24] В. М. Бабич. Алгебра и анализ. 1991. Т. 3. № 5. С. 1-37.

[25] H. Н. Боголюбов, Ю.А. Митропольский. Математические методы в теории нелинейных колебаний. М.: Наука, 1974.

[26] В.И. Арнольд, В. В. Козлов, А. И. Нейштадт. Математические аспекты классической и небесной механики (Итоги науки и техники. Соврем. проблемы математики. Фундаментальные направления. Т. 3). М.: ВИНИТИ, 1985.

[27] В.Ф. Бутузов, А. Б. Васильева. Асимптотические разложения для сингулярно возмущенных уравнений. М.: Наука, 1973.

[28] В. И. Арнольд. Математические методы классической механики. М.: Наука, 1974.

[29] В. Н. ЖКихарев. О необходимых условиях существования и единственности типа решения со слабой распространяющейся особенностью, сосредоточенной в точке, для уравнений гидродинамики в случае двух пространственных переменных. Деп. в ВИНИТИ № В86/8148. М.: ВИНИТИ, 1986. 
[30] J. F. Colombeau. Elementary Introduction to New Generalized Functions. North Holland, 1985.

[31] Ю. В. Егоров. УМН. 1990. Т. 45. № 5. С. 3-40.

[32] В.А. Аркадьев, А.К. Погребков, М.К. Поливанов. ТМФ. 1982. Т. 53. № 2. С. 163-180; 1983. T. 54. № 1. C. 23-37.

[33] V. G. Danilov, G. A. Omel'yanov. Truncation of a Chain Hugoniot-type Conditions for Shock Waves and its Justification for the Hopf Equation. Preprint ESI 502. Vienna: The Ervin Schrödinger Int. Inst. for Mat. Phys., 1997.

[34] R. Ravindran, P. Prasad. Appl. Math. Lett. 1990. V. 3. № 2(3). P. 107-109.

[35] М.И. Вишик, А.В. Фурсиков. Математические проблемы статистической механики. М.: Наука, 1980.

[36] И. А. Кибель, Н.Е. Кочин, Н.В. Розе. Теоретическая гидромеханика. М.: Физматгиз, 1963.

[37] В. Е. Захаров, Е. А. Кузнецов. УФН. 1997. Т. 167. № 11. С. 1137-1167.

[38] Н. Н. Моисеев, В. В. Румянцев. Динамика тела с полостями, содержащими жидкость. М.: Наука, 1965.

[39] В. Ф. Зайцев, А. Д. Полянин. Справочник по обыкновенным диффференциальным уравнениям. Точные решения. М.: Физматлит, 1995.

[40] Л. В. Овсянников. Групповой анализ дифференциальных уравнений. М.: Наука, 1978.

[41] V. Buslaev, A. Grigis. Turning Points for Adiabatically Perturbed Periodic Equations. Preprint Univ. Paris-13, 1998.

[42] А. Д. Брюно. Степенная геометрия в алгебраических и дифференциальных уравнениях. М.: Наука, 1998.

[43] Y. Wang, G. J. Holland. J. Atmos. Sci. 1996. V. 53. P. 411-427.

[44] Л. Н. Гутман. Введение в нелинейную теорию мезоматериальных процессов. Л.: Гидрометеоиздат, 1969.

[45] В. Н. Масленникова, М. Е. Боговский. Математическое моделирование. 1994. Т. 6. № 9. C. $26-32$.

[46] P. G. Saffman. Vortex dynamics. Cambrige: Cambrige Univ. Press, 1992.

[47] J.-L. Lions, R. Temam, S. Wang. Commun. Pure Appl. Math. 1997. V. 1. № 8. P. 707-752.

[48] В. А. Богомолов. Изв. АН СССР. Физика атмосферы и океана. 1979. Т. 15. № 1. С. 29-36.

Поступила в редакцию 29.V.2000 г., после доработки 3.VII.2000 г.

\section{Исправление к статье:}

Н. Илиева, В. Тирринг

"Анионы и дуальность Бозе-Ферми в модели Тирринга при конечных температурах"

(ТМФ. 1999. Т. 121. № 1. С. 40-65)

Ненумерованная формула между (5.10) и (5.11) должна иметь следующий вид:

$$
-\left\langle\left[\Psi_{6 \pi}^{*}(x), \Psi_{6 \pi}\left(x^{\prime}\right)\right]_{\alpha}\right\rangle_{\beta}=\left\langle\left[\Psi_{6 \pi}^{*}(x), \Psi_{6 \pi}\left(x^{\prime}\right)\right]_{+}\right\rangle_{\beta}=-\frac{1}{8 \pi^{2}}\left(\delta^{\prime \prime}\left(x-x^{\prime}\right)-\frac{\pi^{2}}{\beta^{2}} \delta\left(x-x^{\prime}\right)\right) .
$$

